\title{
Spin Orbit Coupling in Orthogonal Charge Transfer States: (TD-)DFT of Pyrene - Dimethylaniline
}

Shivan Bissesar, ${ }^{a}$ Davita M. E. van Raamsdonk, ${ }^{a}$ Dáire J. Gibbons, ${ }^{a}$ René M. Williams ${ }^{a *}$

a Molecular Photonics Group, van 't Hoff Institute for Molecular Sciences (HIMS), Universiteit van Amsterdam, Science Park 904, 1098 XH Amsterdam, Netherlands.

r.m.williams@uva.nl

Keywords: Triplet formation, charge recombination, charge separation, intersystem crossing, SOCTISC, SOCME, electronic coupling.

\begin{abstract}
The conformational dependence of the matrix element for spin-orbit coupling and of the electronic coupling for charge separation are determined for an electron donor-acceptor system containing a pyrene acceptor and a dimethylaniline donor. Different kinetic and energetic aspects that play a role in the spin-orbit charge transfer intersystem crossing (SOCT-ISC) mechanism are discussed. This includes parameters related to initial charge separation and the charge recombination pathways using the Classical Marcus Theory for electron transfer. The spin-orbit coupling, which plays a significant role in charge recombination to the triplet state can be probed by (TD-)DFT, using the latter as a tool to understand and predict the SOCT-ISC mechanism. The matrix elements for spin-orbit coupling for acetone and 4-thio-thymine are used for benchmarking. (Time Dependent-) Density Functional Theory (DFT and TD-DFT) calculations are applied using the quantum chemical program Amsterdam Density Functional (ADF).
\end{abstract}




\section{Introduction}

Using computational chemistry to determine the matrix element for spin orbit coupling ${ }^{1,2}$ (SOCME) in simple organic molecules like acetone, is a challenging task. ${ }^{3}$ SOCME determination in charge transfer systems ${ }^{4}$ goes beyond this, since the transition from a charge transfer state to a local triplet excited state falls within the Marcus theory ${ }^{5,6}$ of electron transfer and can occur in large bifunctional molecules containing an electron donor and an electron acceptor. ${ }^{7,8}$ This process, triplet charge recombination, plays a role in organic photovoltaic materials, heavy-atom-free photosensitizers as well as in LEDs. "Triplet formation by charge recombination is a phenomenon that is encountered in many fields of the photo-sciences and can be a detrimental unwanted side effect, but can also be exploited as a useful triplet generation method, for instance in photodynamic therapy. ${ }^{\prime 9}$ It can also be of importance in a great variety of other applications such as in organic photocatalysis and solar energy harvesting. ${ }^{10,11,12}$ Photosensitizers often contain transition metals ${ }^{13}$ such as $\mathrm{Ru}^{14} \mathrm{Pd}^{15}$ and $\mathrm{Pt}^{16,17}$ In these complexes, intersystem crossing (ISC) is efficient due to spin-orbit interactions, ${ }^{18}$ a relativistic effect usually present in atoms with large nuclei, commonly called the heavy-atom effect. The corresponding ISC is known as spin-orbit intersystem crossing (SO-ISC). ${ }^{19}$ Introducing heavy atoms to form triplet states is far from ideal: increasing cost, low solubility, and not to forget: their environmental impact is significant. The use of heavy-atom free triplet sensitizing dyes is an emerging research field. However, it is still difficult to design these structures with efficient ISC due to a lack of understanding of the relationship between ISC and the molecular structure.

To be able to design efficient heavy-atom free triplet sensitizer molecular systems, knowledge about ISC in these structures should be enhanced. An approach that has been shown to efficiently produce triplet states is by using charge recombination (CR) from a charge transfer (CT) state. In this process, a large orbital angular momentum change is induced by the $\mathrm{CR}$, which can now compensate for the electron spin-flip, to satisfy the rule of angular momentum conservation. This ISC is called spinorbit charge transfer ISC (SOCT-ISC). ${ }^{20}$

Already in 1963, El-Sayed predicted the basis of the SOCT-ISC via his triplet selection rules. ${ }^{21}$ The rate of ISC becomes larger if a radiation-less transition is accompanied by a change of molecular orbital (MO) type, which physically implies that an increased spin-orbit coupling (SOC) between the ${ }^{1} \mathrm{CT}$ and $\mathrm{T}_{1}$ state can result in higher ISC rates. Later, in 1981, the first molecular system undergoing this process was discovered by Okada and colleagues. ${ }^{22}$ Thereafter, Van Willigen suggested that if CR occurs at two aromatic planes, in combination with a large dihedral angle, it can generate the torque needed to spin-flip the electron. ${ }^{23}$ Based on this suggestion, Wasielewski et al. proposed that when the MOs are (nearly) perpendicular to each other, SOCT-ISC is favored over the alternative HFI-ISC (the latter falls outside of the scope of this work, HFI = Hyper Fine Interactions). ${ }^{24}$ Further research 
regarding this observation confirmed the relationship and also found that the polarity of the solvent plays an important role in the rate of ISC. ${ }^{22}$ These observations show that the CT state, as well as the orthogonality of the MOs, is of crucial importance for efficient production of triplets via the SOCT-ISC mechanism. Mataga et al. already stated "that the matrix element of the spin-orbit coupling (SOCME) of the CT state and triplet state is increased in the perpendicular orientation". ${ }^{22}$

The increased interest in this mechanism has not only affected experimental research, but also computational chemists. The use of computational tools to study chemistry problems is emerging and the theory and usability of computational chemistry has reached a level that is advanced enough to study excited state charge transfer processes. ${ }^{9}$ Time-dependent density functional theory ${ }^{25}$ (TD-DFT) calculations can be performed to study the SOCT-ISC mechanism. ${ }^{26}$

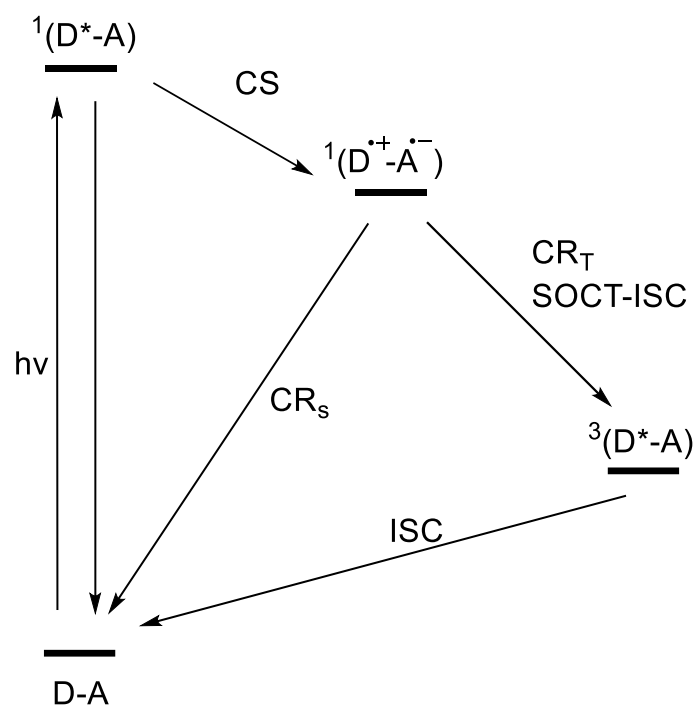

Figure 1. Energy diagram of the photophysical pathways leading to the formation of a triplet state via charge recombination (spin-forbidden $C R$ of ${ }^{1} C T$ to $\left.T_{1}\left(C R_{T}\right)\right)$. $C R_{S}$ is the process from ${ }^{1} \mathrm{CT}$ to the ground state of the electron donor acceptor system (D-A). For clarity: ${ }^{1}\left(D^{*}-A\right)=S_{2},{ }^{1}\left(D^{+}-A^{-}\right)=S_{1},{ }^{3}\left(D^{*}-A\right)=T_{1}$.

The aim of this work is to develop a method within ADF, using TD-DFT, to properly determine the matrix element of spin orbit coupling for charge recombination in electron donor-acceptor molecules, as well as the energetics and conformational effects on this quantity. We will describe the various aspects for one particular molecule ( $\mathrm{N}$-methyl-N-phenyl-1-pyrene-methanamine) that play a role in the decay of charge separated states into local triplet states by the SOCT-ISC mechanism, thereby we generate a framework that can easily be applied to other systems.

For an overview of the SOCT-ISC mechanism, a few clear review papers have been published. ${ }^{9,19,20,27,28}$ To generate triplets via the SOCT-ISC mechanism, three photophysical pathways are involved (see Figure 1): spin-allowed initial charge separation (CS) from $\mathrm{S}_{n} \rightarrow{ }^{1} \mathrm{CT}, \mathrm{CR}$ from the ${ }^{1} \mathrm{CT}$ to the ground state (GS), and spin-forbidden CR from ${ }^{1} \mathrm{CT}$ to $\mathrm{T}_{1}\left(\mathrm{CR}_{\mathrm{T}}\right) \cdot{ }^{26}$ The ultimate goal is to find an optimum between initial charge separation and recombination pathways as discussed by Buck et al. 
They stated in their studies that the spin allowed processes, charge separation $\left(\mathrm{S}_{2} \rightarrow{ }^{1} \mathrm{CT}\right)$ and $\mathrm{CR}$ to the ground state $\left(\mathrm{CR}_{\mathrm{S}}\right)$, are dominant in the yield of the triplet formation. ${ }^{26}$ In order to favor the SOCTISC mechanism, the initial CS and CR pathways should be fine-tuned ${ }^{9}$ by optimizing the SOCME and the electronic coupling.

These aspects that play a role in the SOCT-ISC mechanism can be described via computational methods, however, to our knowledge, a complete clear overview and application of how to use DFT calculations to tackle this complex mechanism was not made before. This work will include the variation of the SOCME $\left(V_{S O C}\right)$, the electronic coupling $\left(V_{C T}\right)^{29}$ and other parameters that are of importance for the formation of triplet states by CR as well as solvent effects (with the conductor-like screening model, COSMO) ${ }^{30}$ that influence the efficiency of the SOCT-ISC mechanism. The radiative lifetime of the triplet state will also be included. The limitations of the computational program, Amsterdam Density Functional (ADF), will be discussed. The aim is to give an overview and application of how to use ADF to describe, understand and predict the SOCT-ISC mechanism.

Within this study, a prototypical molecule, N-methyl-N-phenyl-1-pyrene-methanamine (PyrDMA, see Figure 2), is studied that shows sub-ns charge recombination to the triplet state, as a model system to calculate the various aspects that play a role in the spin-orbit charge transferintersystem crossing (SOCT-ISC) mechanism. PyrDMA is a relatively small molecule, with a short flexible spacer, allowing for the computational study of dihedral angular effects on the SOCT-ISC mechanism.
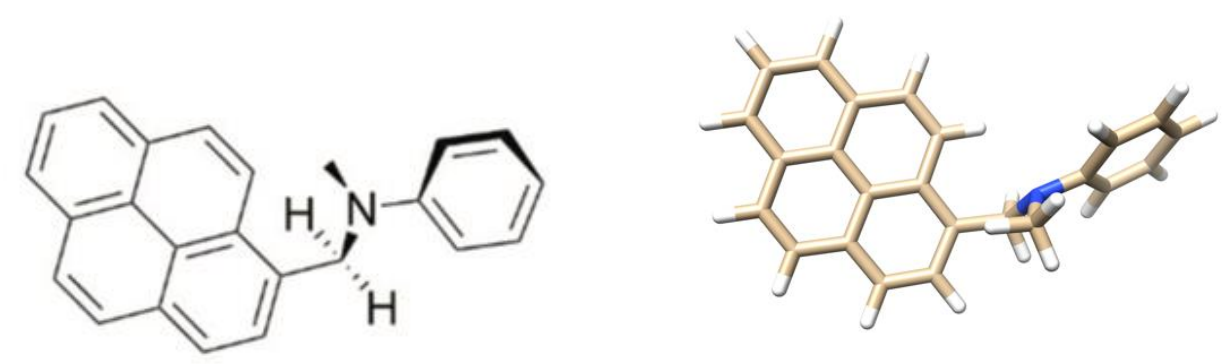

Figure 2. Lewis structure of PyrDMA as well as a 3D representation (Chimera) showing the orthogonality of the donor and acceptor (DFT minimized structure in the ground state). 
The experimentally measured triplet charge recombination rates of PyrDMA in different solvents are indicated in Table 1.

Table 1. Experimentally measured rates for charge recombination to the triplet $\left(k_{C R_{T}}\right)$ in PyrDMA in different solvents. ${ }^{22}$

\begin{tabular}{cc}
\hline Solvent & $k_{C R_{T}}\left(s^{-1}\right)$ \\
\hline NHX & $2.5 \times 10^{10}$ \\
DEE & $4.3 \times 10^{9}$ \\
ACN & $7.0 \times 10^{8}$ \\
NHX $^{*}$ & $2.0 \times 10^{6}$
\end{tabular}

*Intermolecular DMA and Pyrene mixture. Solvents used are n-hexane (NHX), diethyl ether (DEE), acetonitrile (ACN).

The PyrDMA system, containing the text-book chromophore pyrene ${ }^{31}$ and one of the most standard electron donors, dimethylaniline, ${ }^{31,32,33,34}$ shows one of the fastest rates of triplet formation by charge recombination ever measured. Sub-ns charge recombination to the triplet is experimentally observed in $\mathrm{n}$-hexane. The rates for triplet charge recombination vary strongly in the solvents. The charge separation process in PyrDMA and similar molecules occurs on a 1 ps timescale (see later, section on energetics). We set out to quantitatively explain this behaviour and make a roadmap for designing systems with a high triplet generation yield via the SOCT-ISC mechanism.

In this work a nomenclature for the excited states is used that is consistent and directly related to the outcome of the TD-DFT calculations. This often implies that the $S_{1}$ state equals the singlet charge transfer state $\left({ }^{1} \mathrm{CT}\right.$, the lowest state with singlet character of the system). CT character of states will always be specified, as far as possible. The triplet charge transfer state $\left({ }^{3} \mathrm{CT}=\mathrm{T}_{2}\right)$ does not play a role in the photophysics of PyrDMA. ${ }^{22}$ As also discussed in our previous work, ${ }^{9}$ the conversion of the ${ }^{1} \mathrm{CT}$ into the ${ }^{3} \mathrm{CT}$ is expected to occur on a $10 \mathrm{~ns}$ timescale. However, computational results regarding the ${ }^{3} \mathrm{CT}$ are presented when available. 


\section{Experimental: Computational Methods.}

Structures were optimized and properties were calculated with the ADF DFT package (SCM, version 2019) using the default SCF convergence criterion $\left(1 \times 10^{-6}\right)$ on the Lisa cluster of SURFsara. ${ }^{35,}{ }^{36}$ As a starting structure PyrDMA was build and optimized in SPARTAN. All geometry optimizations were conducted using the zero-order regular approximation (ZORA) for relativistic effects. ${ }^{37}$ ADF basis sets of triple zeta plus polarization (TZP or TZ2P) were used (as specified), with no frozen core. Representative ADF input and output files are provided as well as an extensive description of the procedure with (repeated) references (see Supporting Information). Coordinate files for the various excited states are supplied as mol2 files. The results were visualized with UCSF Chimera. ${ }^{38}$ 


\section{Results and Discussion}

\section{Benchmarking the SOCME.}

Transitions between singlet and triplet states are forbidden in a non-relativistic framework, however, intersystem crossing from a singlet to a triplet state is possible in the presence of spin-orbit coupling. It is challenging to calculate the SOCME factor in both an effective and accurate way. Using quantum chemistry, it is possible to gain more insight into the SOCME, because it is the result of the spinoperator expressed by its Hamiltonian. ${ }^{9}{ }^{39}$ This spin-orbit Hamiltonian describes the interaction between the spin and orbital motions of an electron and induces singlet and triplet excitations. The coupling of the spin and orbital momenta of the nucleus and electron is described by the Hamiltonian.

$$
\widehat{H}_{S O}=\alpha_{f s}^{2} \sum_{\mu}^{N} \sum_{m}^{n} \frac{z_{\mu}}{r_{m \mu}^{3}} \overrightarrow{L_{m}} \overrightarrow{S_{m}}
$$

$\alpha_{\mathrm{fs}}$ is the fine structure constant. $z_{\mu}$ represents the effective nuclear charge for nucleus $\mu . \vec{L}$ is the orbital momentum and $\vec{S}$ is the spin momentum operator. The distance between the nucleus and electrons is represented by $r_{m \mu}$. This is an effective one-electron operator. The SOC matrix element ${ }^{40,}$ ${ }^{41}$ was computed by considering the three degenerate $T_{1}$ triplet states $(m=0, \pm 1)$.

$$
V_{S O C}=\left\langle S_{1}\left|\widehat{H}_{S O}\right| T_{1}\right\rangle=\sqrt{\Sigma_{m=0, \pm 1}\left\langle S_{1}\left|\widehat{H}_{S O}\right| T_{1}^{m}\right\rangle^{2}}
$$

Calculating the SOCME between the ${ }^{1} \mathrm{CT}$ and $\mathrm{T}_{1}$ state can be performed by using TD-DFT. The SOCME is represented as the waveform root-mean-square average of the three sublevels.

In order to test that our method (ADF), the functionals and basis sets and are appropriate it is important to perform benchmark calculations. ${ }^{3}$ The first step in this work was therefore to benchmark the method for the SOC matrix $\left(V_{S O C}\right)$ determination, for which acetone and 4-thio-thymine were used as reference molecules. ${ }^{3}$ The SOC matrices for these molecules were previously calculated in a benchmark study. ${ }^{3}$ The SOCME $\left(V_{S O C}\right)$ for these molecules was calculated with different exchange correlation functionals (see Table 2). Using the ground state geometry results in much lower values for $V_{S O C}$. Therefore, the calculations were done at the optimized excited state $\mathrm{S}_{1}$ geometry (PBE exchange correlation/TZP basis). 
The $V_{S O C}$ literature values for 4-thio-thymine lie within the range $138-206 \mathrm{~cm}^{-1}$, while the values for acetone lie within $44-88 \mathrm{~cm}^{-1}$ depending on different computational approaches, exchange correlation (XC) functionals and basis sets. ${ }^{3}$ The SOCME values are benchmarked with ADF using different exchange functionals.

Table 2. The $V_{S O C}$ values for acetone, 4-thio-thymine and PyrDMA calculated with different exchange correlation functionals at the $S_{1}$ geometry (PBE/TZP).

\begin{tabular}{cccccc}
\hline XC & $\begin{array}{c}V_{\text {SOC }}\left(\mathrm{cm}^{-1}\right) \\
\text { Acetone }\end{array}$ & $\begin{array}{c}V_{\text {SOC }}\left(\mathrm{cm}^{-1}\right) \\
\text { 4-thio-thymine }\end{array}$ & \multicolumn{3}{c}{$\begin{array}{c}V_{\text {SOC }}\left(\mathrm{cm}^{-1}\right) \\
\text { PyrDMA }\end{array}$} \\
& Gas & Gas & Gas & NHX & ACN \\
\hline PW91 & 61.85 & 167.50 & 2.68 & 2.75 & 2.85 \\
BLYP & 62.56 & 169.87 & 2.69 & 2.76 & 2.86 \\
CAM-B3LYP & 62.20 & 156.11 & 2.39 & $\mathbf{2 . 4 7}$ & $\mathbf{2 . 5 6}$ \\
CAMY-B3LYP & 62.30 & 138.14 & 2.36 & $\mathbf{2 . 4 1}$ & 1.96 \\
RPBE & 61.00 & 164.18 & 2.63 & 2.69 & 2.79 \\
HTBS & 61.29 & 166.56 & 2.66 & 2.73 & 2.83 \\
S12y & 60.53 & 160.90 & 2.59 & 2.66 & 2.76 \\
LB94 & 67.76 & 181.70 & 3.15 & 3.22 & 3.34 \\
KT1 & 59.55 & 156.83 & 2.59 & 2.65 & 2.75 \\
BhandH & 60.61 & 103.83 & $\mathbf{2 . 0 8}$ & $\mathbf{2 . 1 3}$ & 1.74 \\
\hline
\end{tabular}

$X C$ is the density functional. The geometry optimisations of the $S_{1}$ state were calculated using a TZP basis and PBE XC functional. A TZP basis was used for calculating the SOC. Note the state shift for 4-thio-thymine and the CAM(Y)-B3LYP functionals. Highest values are found for LB94. Values in bold are for $S_{1}-T_{1}$ transitions the rest are $S_{1}-T_{2}$.

The exchange correlation functionals result in $V_{S O C}$ values of $61.97 \pm 2.24 \mathrm{~cm}^{-1}$ for acetone and 156.62 $\pm 21.67 \mathrm{~cm}^{-1}$ for 4-thio-thymine (see Table 2 ). The exchange correlation functional doesn't significantly affect the SOCME value, as expected. All $V_{S O C}$ values except for the one which uses BhandH lie in the range from literature. It can be noted that the highest values are found for the LB94 exchange correlation. It can be noted that we do this benchmark study relative to the reported work ${ }^{3}$ on other computational methods. We do not claim comparison to precise experimental data.

In range-separated hybrid (RSH) DFT methods the amount of exact exchange increases with the electron-electron distance. In many RSH approximations, this is used to restore the correct longrange asymptotic behaviour of the corresponding potential. ${ }^{42}$ Their proper asymptotic behaviour renders RSH methods potentially more accurate for the description of electronic excitations. The RSH 
functionals CAM-B3LYP and the CAMY-B3LYP functionals correctly predict the SOCME values and also predict the correct matrix element $V_{S O C}$ with the state shift in 4-thio-thymine. This is especially important for describing CT states. ${ }^{43}$ The CAM-B3LYP however, cannot be used in geometry optimisations with ADF. Therefore, the calculations for the geometry optimizations are performed with TD-DFT with the range-separated CAMY-B3LYP functional (next to the PBE functional, that was also used). The CAMY-B3LYP functional makes use of a range separation parameter $\gamma$, which is the inverse distance and measures how fast range separation switches from short range to long range. Changing the range separation parameter does not significantly affect the $V_{S O C}$ values for acetone and 4-thiothymine (see Figure 3). For PyrDMA, choosing a low range separation parameter leads to higher $V_{S O C}$ value, therefore values 0.34 and 0.1 were used. The former is the default value for the range separated CAMY-B3LYP hybrid (see Figure 3).
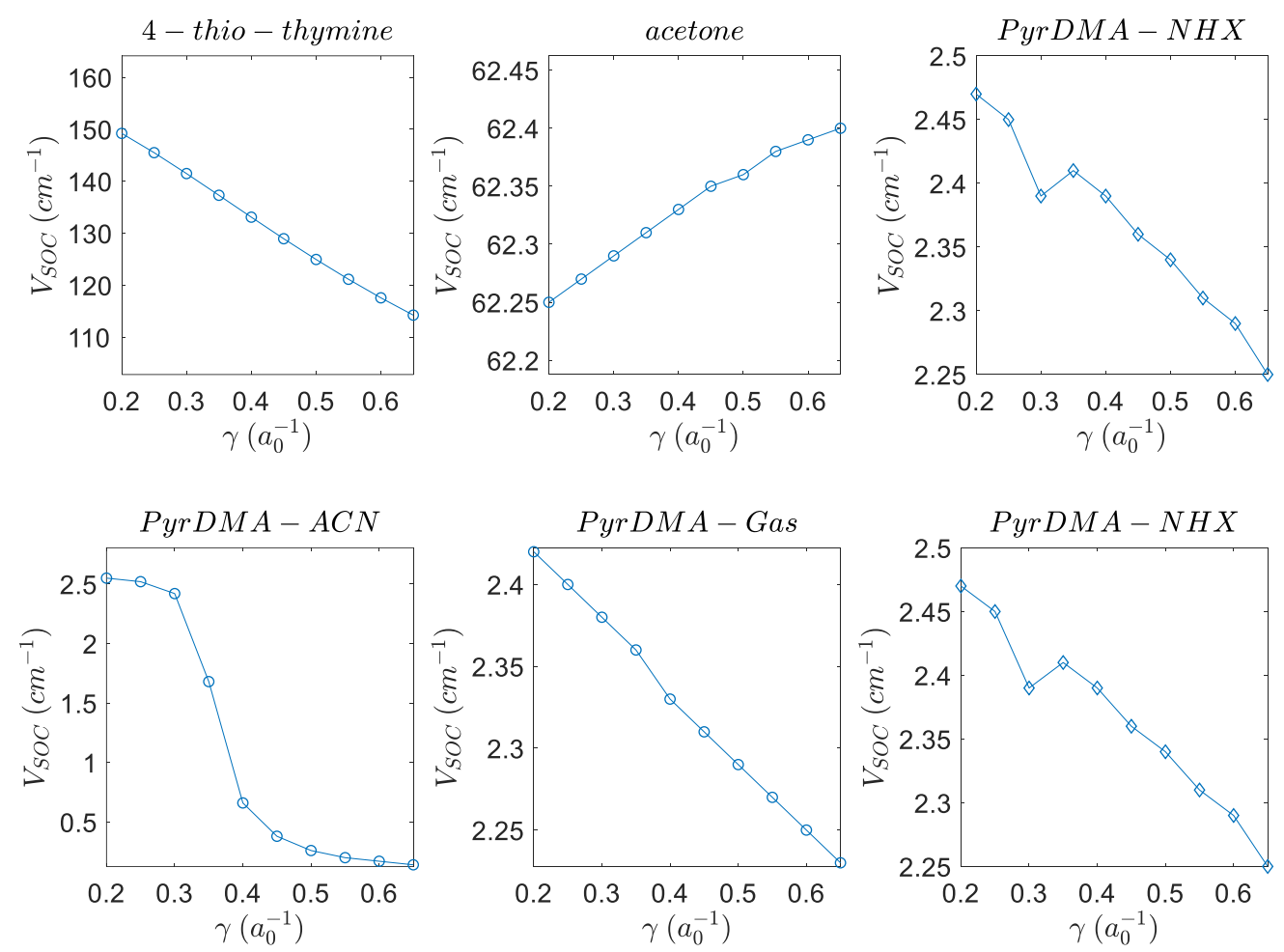

Figure 3. The dependence of $V_{S O C}$ values for 4-thio-thymine, acetone and PyrDMA as function of the range separation parameter $\gamma$ (units of inverse Bohr radius $=52.9117 \mathrm{pm}$ ) in the gas-phase. Two solvents are also shown for PyrDMA. The geometries are calculated with a TZP basis and PBE XC, while the SOC values are calculated using a TZP basis and the CAMY$B 3 L Y P X C$ functional. The behaviour is similar to the results on acetone and 4-thio-thymine from Gao. ${ }^{3}$

\section{Excited state geometries of PyrDMA.}

After benchmarking the SOCME method and assessing the correct XC for charge transfer systems, the next step, in order to study the SOCT-ISC mechanism, is to do geometry optimizations. Geometry 
optimizations are performed for the ground-state (GS or $\left.S_{0}\right)$, and the singlet $\left(S_{n}\right)$ and triplet $\left(T_{n}\right)$ excited states of PyrDMA. The intersystem crossing rate depends rather strongly upon the mutual configuration of donor and acceptor groups as well as the solvent polarity. ${ }^{44}$ Therefore, the optimized geometries of the excited states are calculated in gas-phase (Gas), n-hexane (NHX) and acetonitrile (ACN). Representative geometries are presented in Figure 4 to Figure 6.
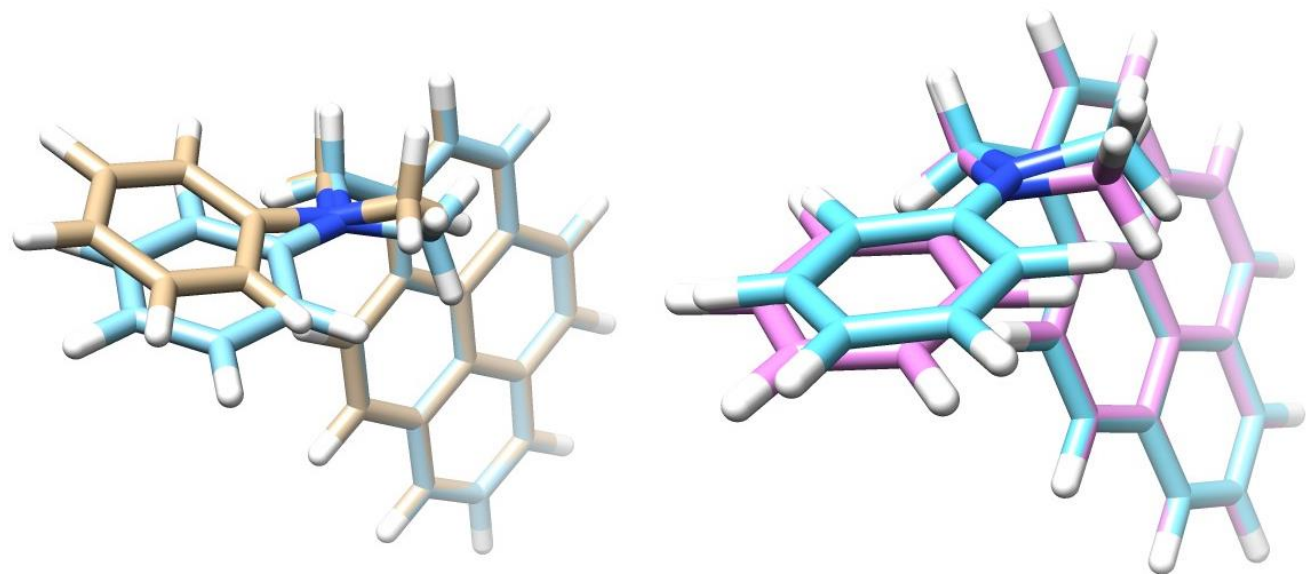

Figure 4. Left: A comparison between the optimized molecular structures of the ground state (in brown) and the $S_{1}$ state (in blue). Right: $A$ comparison between the $S_{1}\left(={ }^{1} \mathrm{CT}\right)$ and the $T_{2}\left(={ }^{3} \mathrm{CT}\right)$ state (in pink). (PBE/TZP)

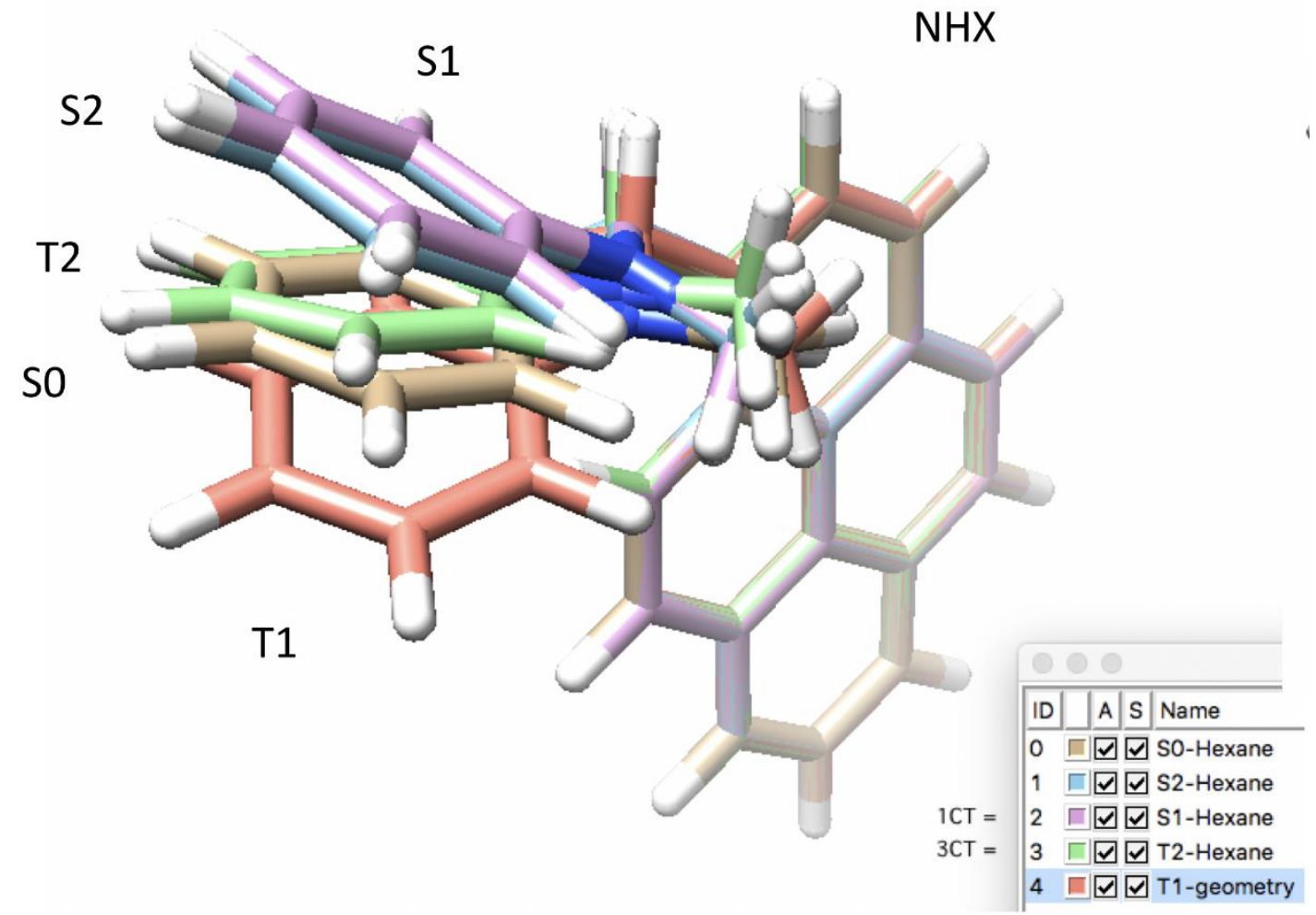

Figure 5. Representation of the five states (PBE/TZP) involved in the photophysical processes of PyrDMA, with overlay of the pyrene unit, clearly shows that the aniline donor group "moves up and down" (relative to the pyrene unit) in going from $S_{0}$, to $S_{2}, S_{1}$ and $T_{1}$ in $n$-hexane solvent. 
It can be noted that $\mathrm{T}_{2}$ (= triplet charge transfer state) does not play a role in the photo-physics. Furthermore, the strongest structural deviation of the triplet state $\left(T_{1}\right)$ is remarkable (see Figure 5 ).

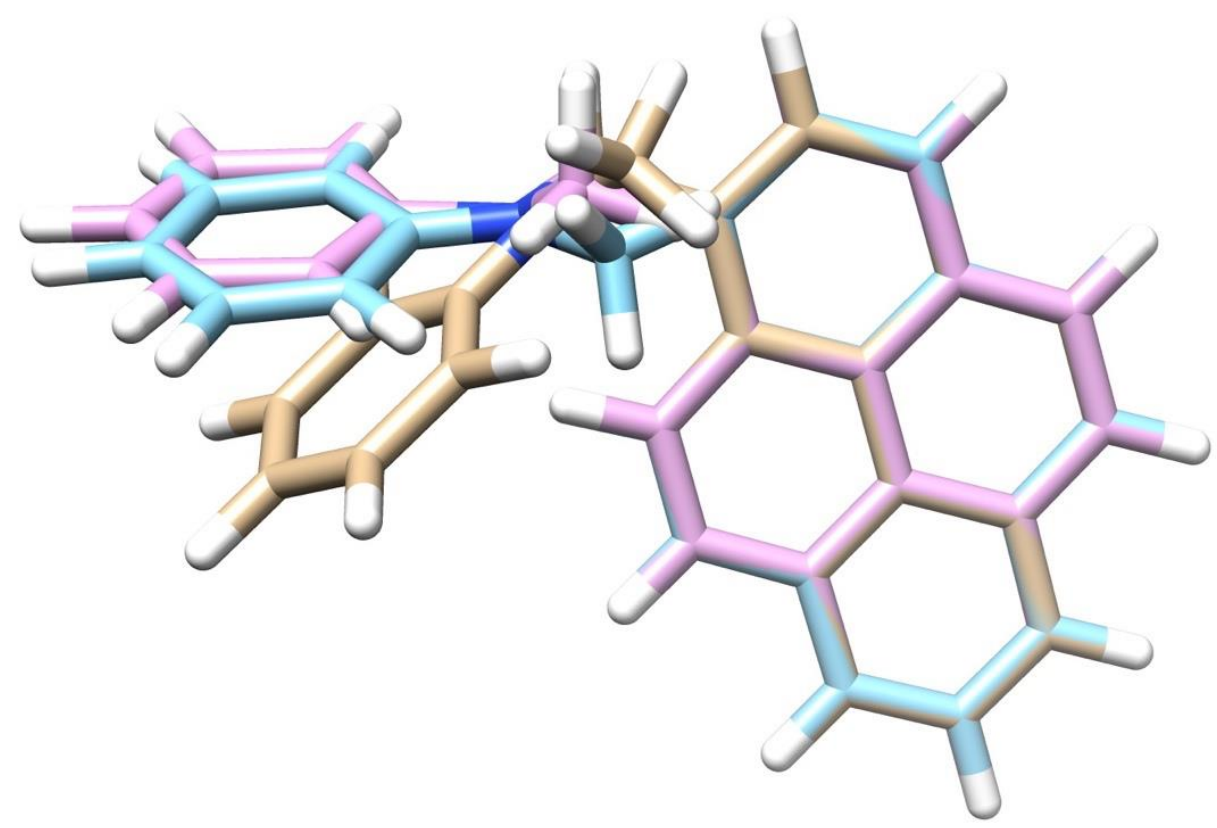

Figure 6. A comparison between the optimized molecular structures of the ${ }^{1} C T\left(=S_{1}\right)$ states in $A C N$ (in brown) in NHX (in blue) and in the gas phase (in pink). Note that the molecular structure of ${ }^{1} \mathrm{CT}$ is strongly influenced by a polar solvent (CAMY$B 3 L Y P / T Z P)$.

The comparisons between the optimized geometries show a strong structural similarity between the $\mathrm{S}_{1}\left({ }^{1} \mathrm{CT}\right)$ and the $\mathrm{T}_{2}\left({ }^{3} \mathrm{CT}\right)$ structure (see Figure 4), which makes physical sense as the $\mathrm{S}_{1}$ and $\mathrm{T}_{2}$ are almost isoenergetic. The arrangement of the atoms around the nitrogen atom in the $\mathrm{S}_{1}$ state is more flat, as compared to the ground state $S_{0}$, in which the nitrogen atom is more pyramidalized. The optimized geometries form a "waving hand" structure (see Figure 5), which moves up and down with the excited states. Energetic and structural information of the various excited states and their geometries is represented in Table 3 to Table 5. 
Table 3. The total bonding energies are calculated at the optimized geometries of the states with the TZ2P basis and the CAMY-B3LYP functional with the default parameters for range separation.

\begin{tabular}{cccccc}
\hline Solvent & $\mathrm{E}_{\mathrm{TB}} \mathrm{S}_{0}(\mathrm{eV})$ & $\mathrm{E}_{\mathrm{TB}} \mathrm{S}_{2}(\mathrm{eV})$ & $\mathrm{E}_{\mathrm{TB}} \mathrm{S}_{1}(\mathrm{eV})$ & $\mathrm{E}_{\mathrm{TB}} \mathrm{T}_{2}(\mathrm{eV})$ & $\mathrm{E}_{\mathrm{TB}} \mathrm{T}_{1}(\mathrm{eV})$ \\
\hline Gas & -389.543 & -385.636 & -386.151 & -386.020 & -387.306 \\
$\mathrm{NHX}$ & -389.612 & -385.839 & -385.817 & -386.070 & -387.375 \\
$\mathrm{ACN}$ & -389.700 & -386.062 & -386.290 & -386.153 & -387.455 \\
\hline
\end{tabular}

Table 4. The differences in total bonding energies are calculated at the optimized geometries of the states with the TZ2P basis and the CAMY-B3LYP functional with the default parameters for range separation, relative to the ground state in the solvent.

\begin{tabular}{cccccc}
\hline Solvent & $\Delta \mathrm{E}_{\mathrm{TB}} \mathrm{S}_{0}$ & $\Delta \mathrm{E}_{\mathrm{TB}} \mathrm{S}_{2}$ & $\Delta \mathrm{E}_{\mathrm{TB}} \mathrm{S}_{1}$ & $\Delta \mathrm{E}_{\mathrm{TB}} \mathrm{T}_{2}$ & $\Delta \mathrm{E}_{\mathrm{TB}} \mathrm{T}_{1}$ \\
& $(\mathrm{eV})$ & $(\mathrm{eV})$ & $(\mathrm{eV})$ & $(\mathrm{eV})$ & $(\mathrm{eV})$ \\
\hline Gas & 0 & 3.907 & 3.392 & 3.523 & 2.237 \\
$\mathrm{NHX}$ & 0 & 3.773 & 3.795 & 3.542 & 2.237 \\
ACN & 0 & 3.638 & 3.410 & 3.547 & 2.245 \\
\hline
\end{tabular}

In Table 3 and

Table 4 information on the total bonding energy at the optimized geometries is represented, as output energies as well as energy difference relative to the ground state. Total bonding energy does not take into account excitation energies, but consists of several contributions: electrostatic energy, kinetic energy, Coulomb (steric + orbital interaction) energy, XC energy and solvation (see ADF manual for further information). Optimized ground and excited state geometries vary with solvent, but the total bonding energy difference between e.g. the ground state and the local pyrene triplet state $\left(T_{1}\right)$ is consistent. In Table 5, we indeed observe the "up down" movement of the excited states to form the molecular fin.

Table 5. The dihedral angle $\theta$ of the optimized geometries calculated with the TZ2P basis and the CAMY-B3LYP functional with the default parameters for range separation. See also Figure 8.

\begin{tabular}{cccccc}
\hline Solvent & $\mathrm{S}_{0}$ & $\mathrm{~S}_{1}$ & $\mathrm{~S}_{2}$ & $\mathrm{~T}_{1}$ & $\mathrm{~T}_{2}$ \\
\hline Gas & 67.0 & 69.9 & 65.7 & 65.8 & 64.0 \\
$\mathrm{NHX}$ & 66.2 & 65.7 & 67.1 & 65.8 & 63.9
\end{tabular}




$\begin{array}{llllll}\mathrm{ACN} & 66.4 & 71.6 & 70.4 & 66.4 & 64.1\end{array}$

Following the El Sayed rules, the ISC rate becomes higher if a radiation-less transition is accompanied by a change of molecular orbital (MO) type as previously mentioned. Thus, an increased spin-orbit coupling (SOCME) between the ${ }^{1} \mathrm{CT}$ and $\mathrm{T}_{1}$ state results in higher ISC rates. When the donor and acceptor are in an orthogonal orientation, there will be a change in orbital angular momentum, which will induce a large SOCME, allowing for $\mathrm{CR}_{\mathrm{T}}$. Studying the HOMO (-) and LUMO (+) MOs can give insight into whether or not a large SOCME can be expected for the system. Multiple MOs may contribute equally to an excited state and can be transformed to a Natural Transition Orbital (NTO) as shown in Figure 7. With the NTO, the qualitative description of the electronic transition can be simplified and it gives insight into the localization of excitations.

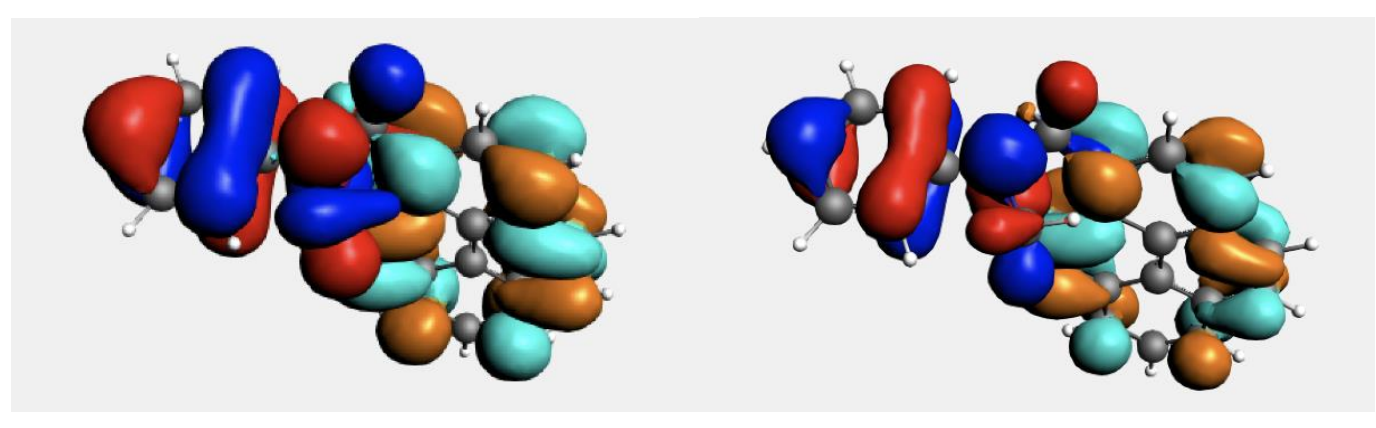

Figure 7. The natural transition orbitals (NTOs) for the singlet ${ }^{1} \mathrm{CT}$ (left) and triplet ${ }^{3} \mathrm{CT}$ (right) charge transfer states (PBE/TZP), SS NTO-1, $2.867 \mathrm{eV}, S_{1}={ }^{1} \mathrm{CT}$ and ST NTO-1, $2.854 \mathrm{eV}, T_{2}={ }^{3} \mathrm{CT}$.

The NTOs ${ }^{45}$ show a clear charge transfer transition between the HOMO of the aniline (red-blue) to the LUMO on the pyrene (brown-cyan). There is a strong similarity between the singlet and triplet charge transfer state. The energy difference $(13 \mathrm{meV})$ indicates a J value of $6.5 \mathrm{meV}$.

\section{Excitation energies and scheme at optimized geometries.}

The excitation energies increase in the order $\mathrm{S}_{0}, \mathrm{~T}_{1}, \mathrm{~T}_{2}, \mathrm{~S}_{1}$ and $\mathrm{S}_{2}$. From the order of excitation energies of PyrDMA, the following conclusions can be drawn about the states:

$\mathrm{S}_{2}$ is the locally excited (LE) state of pyrene, from which charge transfer occurs. The numerical results match quite well with the experimentally measured singlet excited state of pyrene $E_{S_{2}}=3.26 \mathrm{eV} .{ }^{63,64}$ 
$\mathrm{S}_{1}$ state is the charged separated singlet charge transfer state ${ }^{1} \mathrm{CT}$. The $\mathrm{S}_{1}$ state has an excitation energy near $E_{S_{1}}=2.87 \mathrm{eV}$.

$\mathrm{T}_{2}$ state is the triplet charge transfer state ${ }^{3} \mathrm{CT}$, which has about the same excitation energy as the singlet charge transfer state. The triplet charge transfer state does not play a role in the photophysical processes, since the lifetime of the ${ }^{1} \mathrm{CT}$ state is only $40 \mathrm{ps}$, as determined experimentally. The proton hyperfine interactions proceed with a rate of about $8 \times 10^{7} \mathrm{~s}^{-1} .^{9}$

$\mathrm{T}_{1}$ is final triplet product state, which can again decay to the ground state $\mathrm{S}_{0} \cdot{ }^{46}$ The excitation energy of the triplet state matches well with the experimental triplet excitation of pyrene. For a better overview of the scheme, see Figure $14.65,66$

\section{Angular dependence of Spin Orbit Coupling and of the Electronic Coupling}

The electronic coupling ${ }^{47}$ and SOCME values ${ }^{48}$ need to be calculated at different nuclear coordinates ${ }^{49}$ in order to determine their angular dependence. The dihedral angle defined in Figure 8 is the nuclear coordinate which is varied in this study.
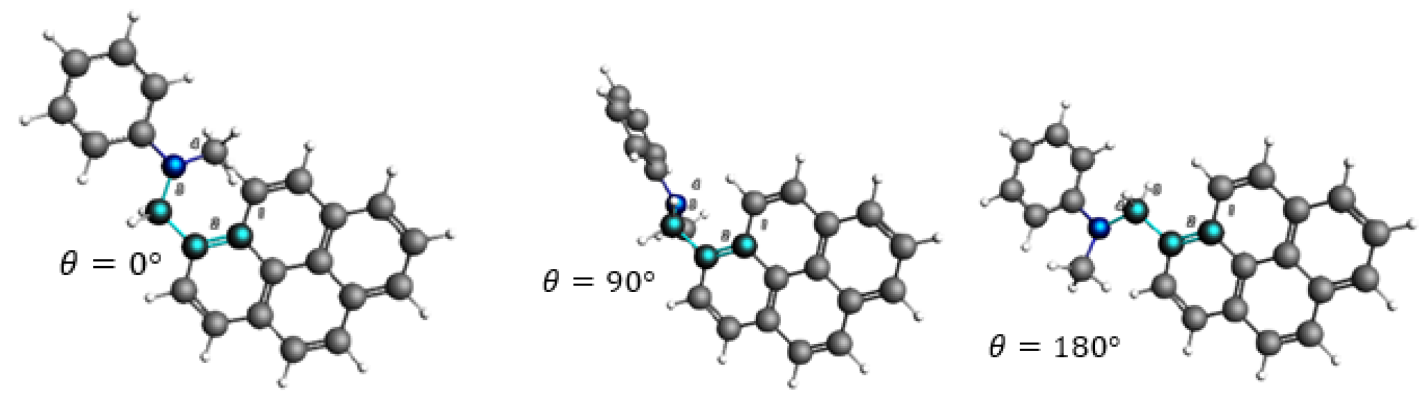

Figure 8. The dihedral angle defined between the pyrene acceptor and the dimethylaniline donor. Left: The ground state of PyrDMA at a dihedral angle of $0^{\circ}$. Centre: Ground state of PyrDMA at an orthogonal geometry. Right: The molecule at a dihedral angle of $180^{\circ}$.

Different parameters are needed for the Marcus theory and the electronic coupling is one of them. The electronic coupling facilitates charge separation (CS). CS can be visualized by studying the molecular orbitals and their electronic transitions. For example, the electronic transition from a frontier HOMO on the donor-unit to the LUMO of the acceptor molecule results in a charge-separated state. ${ }^{50}$ The electronic coupling is the same as the effective charge transfer integral $\left(V_{C T}\right) \cdot{ }^{51}$ The following equation shows the correlation of the charge transfer integral with the transfer integral $J$, overlap integral $S$, and site energy $\varepsilon$.

$$
V_{C T}=\frac{J-\left(\frac{s(\varepsilon 1+\varepsilon 2)}{2}\right)}{1-s^{2}}
$$


The charge transfer integral corresponding to the electronic coupling between the LE state and the CTstate can be calculated with the quantum chemical program ADF. The charge transfer integrals are calculated using a fragment approach (see Figure 9). The fragment approach makes use of MOs on the individual fragmental molecules as a basis set of the calculations on a system containing two or more fragment molecules. ${ }^{52}$ The aniline donor and pyrene acceptor are split and form two fragments. The $\mathrm{CH}_{2}$ group is deleted, and hydrogen atoms are added to the free valences. The two fragments (pyrene and $\mathrm{N}-\mathrm{H}$-methylaniline) are first computed separately, followed by a computation that calculates the interaction between the two fragments. The charge transfer integrals, site energies, and overlap integrals between the two fragment orbitals are calculated. The electronic coupling is calculated as a function of the dihedral angle between pyrene and N-H-methylaniline (see Figure 9) at the geometry of the precursor state, in this case the $S_{2}$.

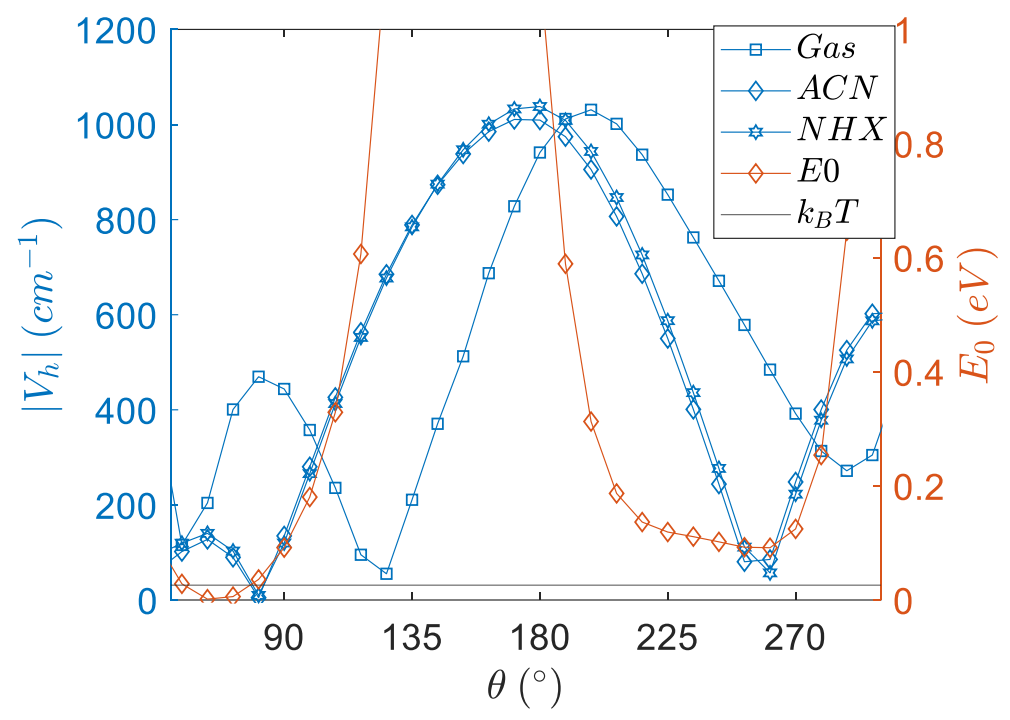

Figure 9. The electronic coupling as function of the dihedral angular coordinate. The calculations are performed starting with the $S_{2}$ geometry in different solvents (PBE/TZP). Black vertical lines indicate the angles at the optimized $S_{2}$ geometries. The electronic coupling is calculated with a TZP basis and the CAM-B3LYP XC functional using the fragment method. The ground state energy $\left(E_{0}\right)$ in $N H X$ of the intact molecule alongside the thermal energy $\left(k_{B} T\right)$ is plotted to indicate the important range of conformations for the whole intact PyrDMA molecule. The black horizontal line represents the thermal energy available in the ground state. Note that the $x$-axis ranges from 45 to 300 degrees.

Figure 9 clearly shows that optimal charge transfer interaction can occur at $\sim 180^{\circ}$. Around $0^{\circ}$ and $360^{\circ}$ the $V_{C T}$ values also rise, but due to interactions between the $\mathrm{N}$-methyl group and a $\mathrm{H}$ atom of pyrene, steric repulsive interactions dominate (see total bonding energies). The $E_{0}$ shows that the preferred conformation in the ground state is characterized by a dihedral angle of about $70^{\circ}$. 
The SOCME $\left(V_{S O C}\right)$ values are required for the charge recombination rates in the SOCT-ISC mechanism. Studying an interaction between two states is normally studied from the precursor state, which implies that the SOCME values should be calculated at the $\mathrm{S}_{1}$ geometry. From a Potential Energy Surface Scan (PESScan), the SOCME values of the singlet charge transfer state $\left(\mathrm{S}_{1}-\mathrm{T}_{1}\right.$ matrix element) were computed as a function of the dihedral angle of the optimized $\mathrm{S}_{1}$ geometry in different solvents.

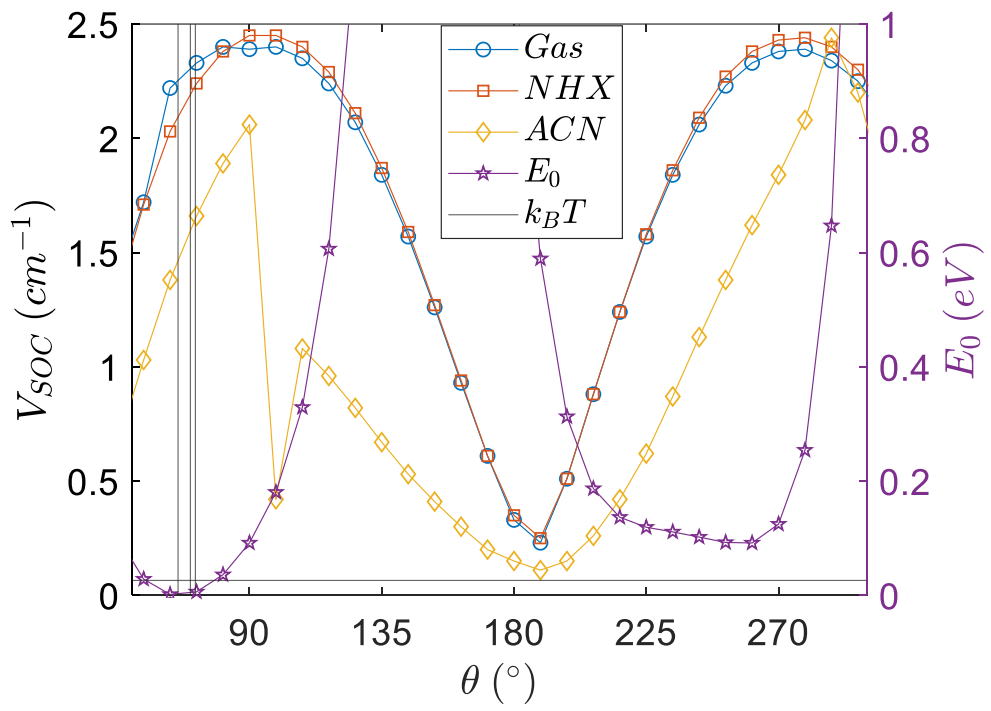

Figure 10. The SOCME values for the singlet charge transfer state ${ }^{1} \mathrm{CT}$ as function of dihedral angle starting with the optimized $S_{1}$ geometry (TZP/PBE) in different solvents for the whole intact PyrDMA molecule. Weak solvent effects are observed for $n$ hexane and the gas-phase, however the dependence is modulated in ACN. Black vertical lines indicate the angles at the optimized geometries of the $S_{1}$ state. The quantities are calculated with the TZP basis and the CAMY-B3LYP functional at its default parameters. The ground state energy in $N H X\left(E_{0}\right)$ of the intact molecule alongside the thermal energy $\left(k_{B} T\right)$ is plotted to indicate the important range of conformations. Note that the $x$-axis ranges from 45 to 300 degrees.

In Table 6, the values of the electronic coupling, the spin orbit coupling at optimized geometries with this method as well as at their maxima, are given. This gives an overview of the range of angles and couplings that can be considered. The electronic coupling and the SOCME curves are combined in Figure 11, in which the squared quantities (proportional to the optimal rates) are displayed.

\begin{tabular}{lrrr}
\hline & \multicolumn{1}{l}{ Gas } & \multicolumn{1}{l}{$\mathrm{NHX}$} & \multicolumn{1}{c}{$\mathrm{ACN}$} \\
\hline$V_{C T, S 2}\left(\mathrm{~cm}^{-1}\right)$ & 263.11 & 123.24 & 96.40 \\
$V_{S O C, S 1}\left(\mathrm{~cm}^{-1}\right)$ & 2.36 & 2.41 & 1.95 \\
$\theta_{C T, \text { max }}$ & 197.98 & 179.99 & 171.00 \\
$V_{C T, \text { max }}\left(\mathrm{cm}^{-1}\right)$ & 1031.00 & 1037.90 & 1010.70 \\
$\theta_{S O C, \text { max }}$ & 81.06 & 90.05 & 89.90 \\
$V_{S O C, \text { max }}\left(\mathrm{cm}^{-1}\right)$ & 2.40 & 2.45 & 2.06 \\
\hline
\end{tabular}


Table 6. The spin orbit and electronic coupling at the optimized geometries. The value of the maximum SOC value near an orthogonal geometry and the maximum electronic coupling near $180^{\circ}$. The geometries are calculated with TZP and the CAMYB3LYP functional.
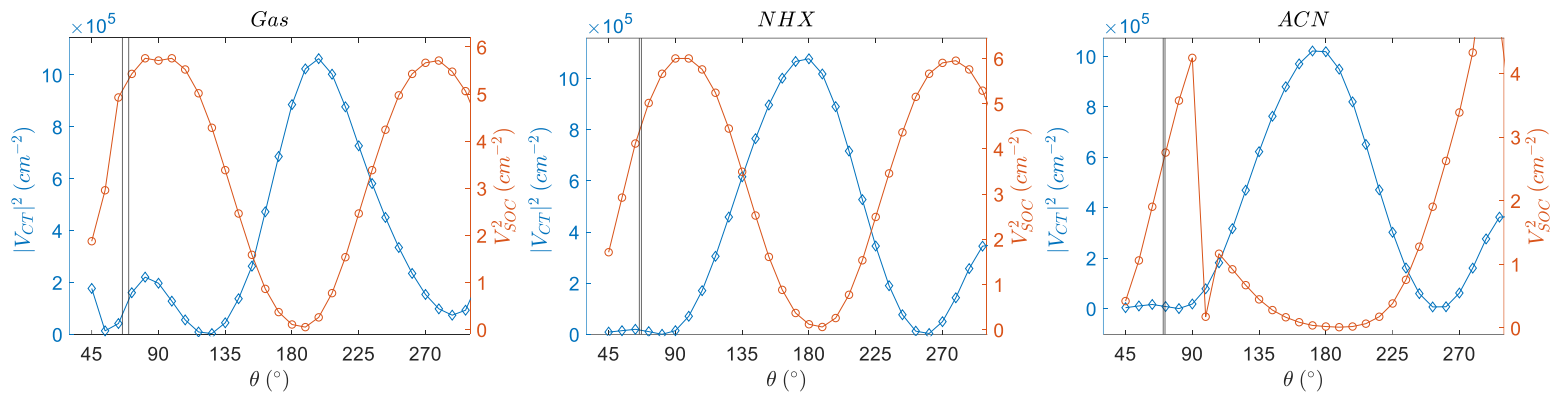

Figure 11. The conformational dependence of the electronic coupling and the SOCME for the singlet charge transfer state. The $V_{S O C}$ and $V_{C T}$ curves show complementary behaviour. The black vertical lines are the dihedral angles at the optimized $S_{1}$ and $S_{2}$ geometry.

The singlet charge transfer state ${ }^{1} \mathrm{CT}$ shows slower SOCT-ISC at dihedral angles near $180^{\circ}$ in the solvents. The dihedral angle for optimal SOCT-ISC is around $90^{\circ}$. The SOCME values become enhanced in this near perpendicular configuration, which has previously been observed. ${ }^{22}$ Mataga et al. stated that "the matrix element of the spin-orbit coupling (SOCME) of the CT state and triplet $\left(T_{n}\right)$ state is increased in the perpendicular orientation". ACN is highly polar solvent and thus has more significant impact on the geometry of the dipolar charge transfer state $S_{1}$ state as compared to $n$-hexane and the gas-phase. This leads to a modulated SOCME curve for ACN. Furthermore, the ACN curve shows a discontinuous behaviour, which can be explained by an electronic effect related to charge stabilisation of the dipolar charge transfer state in ACN (see next section) and is correlated to a reduced distance between the charges.

The conformational dependence of the electronic coupling behaves complementary to the SOCME curves, which is in line with the current scientific paradigms. The electronic coupling near $180^{\circ}$ in the solvents is high, which suggests that charge separation can occur very rapidly near this conformation. The orthogonal orientation of the $\pi$-type MOs between the donor and acceptor favours the SOCT-ISC mechanism, however, it is not favourable for electron transfer, due to the diminished electronic coupling in this configuration. This substantiates that the electronic coupling diminishes with 
a dihedral angle going towards orthogonality. At the optimized geometries, there is lower charge separation and a higher charge recombination rate.

Even though the angular dependence of charge transfer integrals has been reported before, ${ }^{53,54}$ we believe that the combination of calculating the spin-orbit coupling matrix element $\left(V_{S O C}\right)$ and the electronic coupling $\left(V_{C T}\right)$ for one molecule is unprecedented. It has to be highlighted that the total energies $\left(E_{0}\right)$ in figure 9 and 10 indicate that the conformation of PyrDMA is rather restricted and that for instance the range of angles between 120 and 190 degrees is totally unaccessible for the molecule. It has to be realized that the curves in figure 9 and 10 are hypothetical curves obtained by forcing the molecule in certain conformations that it normally would not or could not attain. This is the only way to determine the angular dependence of these quantitities.

\section{Angular dependence of the charge transfer character, electron hole distance and energetics}

Next to the spin-orbit coupling matrix element $\left(V_{S O C}\right)$ and the electronic coupling $\left(V_{C T}\right)$, other properties as a function of the dihedral angular coordinate can be probed, like CT character and the distance between the centres of charge ${ }^{55}$ (see Figure 12). The method for charge transfer descriptors is based on that of Plasser, Lischka et al. ${ }^{56}$ The NTO and DESCRIPTORS key words allow for the calculation of $R_{H E}$, the average distance relating to the hole-electron separation upon electronic excitation. A large distance can be found for CT excitations and a short distance for valence excitations. The charge transfer descriptor gives insight into the charge-transfer character, which has a value between 0 and 1 . A value of 0 represents a locally excited state with no CT, and 1 of a totally charge separated state.
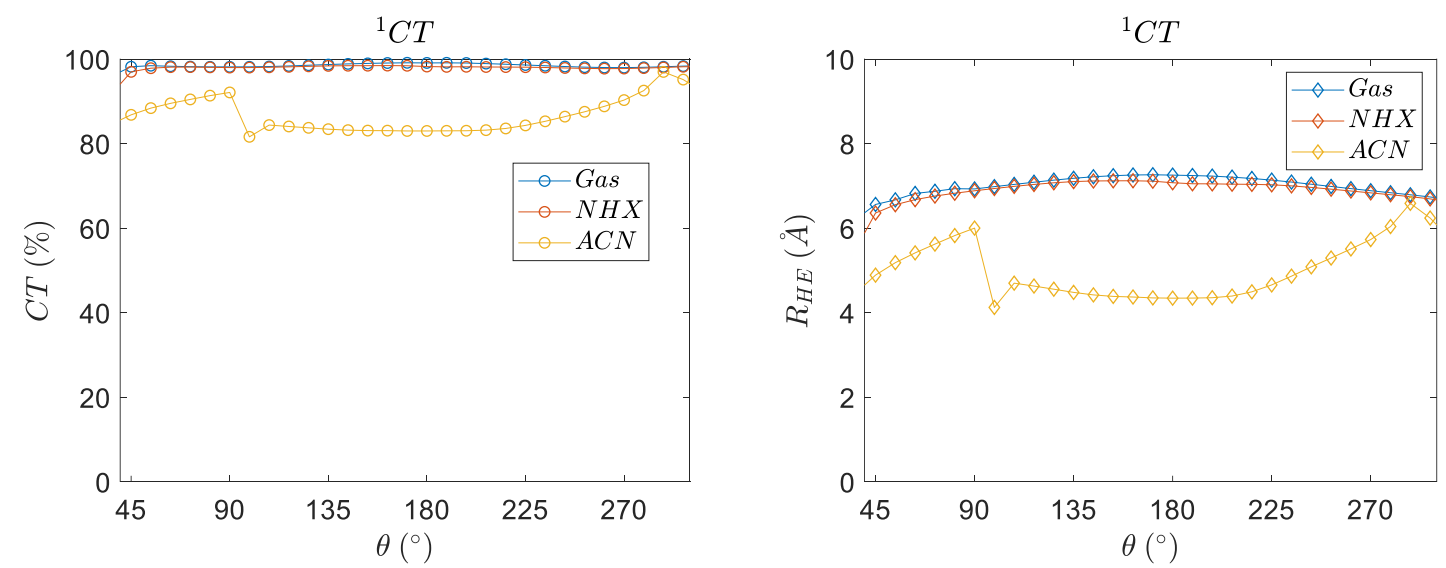

Figure 12. The conformational dependence of the charge transfer character. Charge transfer descriptors for the ${ }^{1} \mathrm{CT}$ singlet charge transfer state calculated starting with the $S_{1}$ geometry. The descriptors are calculated as function of the dihedral angle at the optimized singlet charge transfer state $S_{1}$ in different solvents. Geometries are calculated with PBE and TZP. The quantities are calculated with the TZP basis and the CAMY-B3LYP functional. 
The singlet charge transfer states show near $98.0 \%$ charge transfer character in gas-phase and in nhexane, while a lower charge transfer character is observed in ACN. The charge transfer state has, on average, a lower charge transfer of approximately $86.6 \%$ in ACN. The electron hole distance is approximately constant near $6.25 \AA$. The electron hole distance does not significantly change for the singlet and triplet charge transfer states in gas and n-hexane (see Appendix IV).

The abrupt change in distance in ACN is correlated to the changes in the SOCME value as observed in Figure 13, which suggests that this discontinuity is not a 'error'. ${ }^{57}$ The distance shows a significant change in $\mathrm{ACN}$, which most likely is related to an electronic effect induced by coulombic charge stabilisation of the charge transfer state in the highly polar ACN solvent, accompanied by a structural contraction. Measuring the centre to centre distance with the optimized geometries agrees with this interpretation, as a slightly contract structure is present in ACN solvent (see Table 7).

Table 7. The centre to centre distance between pyrene and DMA. The quantity is calculated by taking the average distance between the furthest hydrogen atoms and closest carbon atoms between the donor and acceptor (excluding spacer atoms). The geometries are calculated with the TZ2P basis and CAMY-B3LYP exchange correlation.

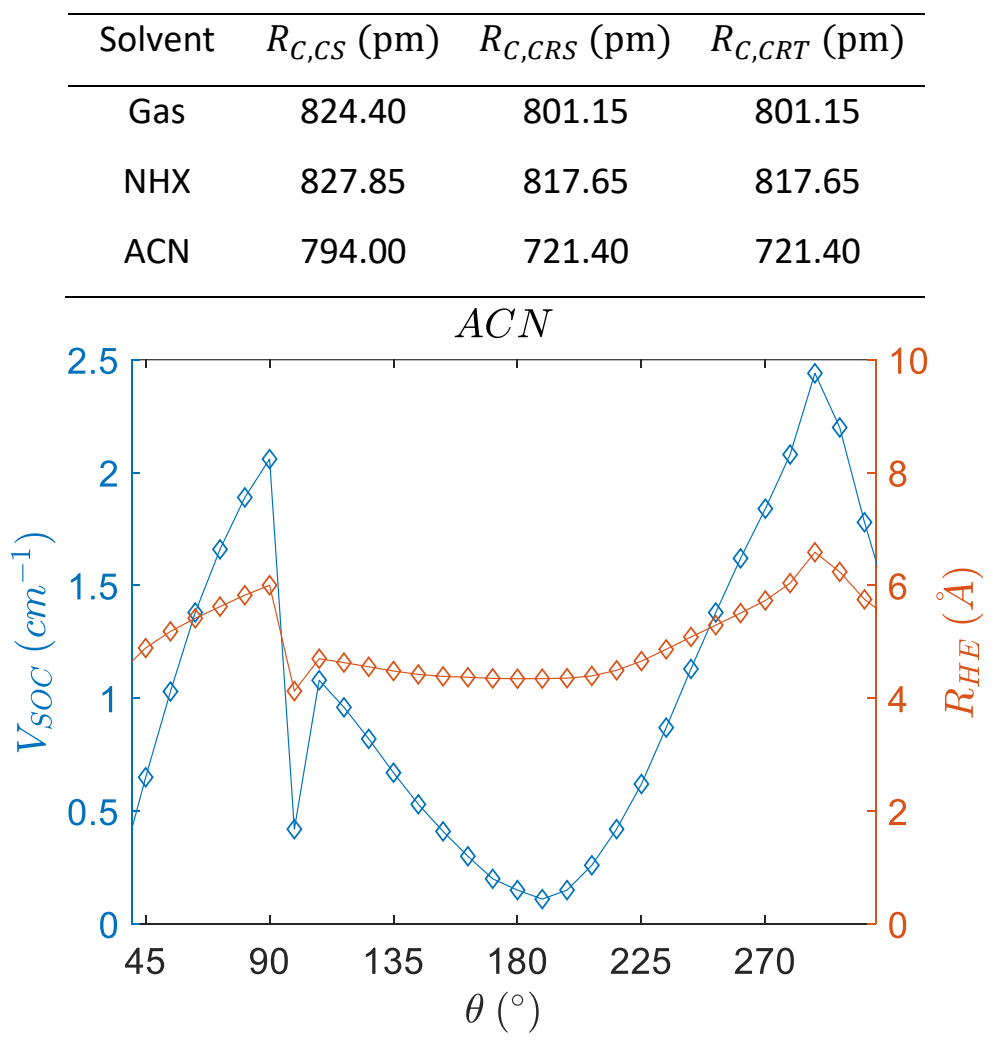

Figure 13. The correlation between the SOCME value and the electron hole distance in ACN. Geometries are calculated with $P B E$ and TZP. The quantities are calculated with the TZP basis and the CAMY-B3LYP functional. 
Figure 13 shows that, at a dihedral angle of $\sim 95^{\circ}$, the SOCME and the electron-hole separation distance show a minimum, and both show a maximum at $\sim 290^{\circ}$. The reduced $R_{H E}$ values correlate with a structural change and reduced distance between donor and acceptor. 


\section{Energetic and kinetic considerations for charge separation and recombination pathways}

In this section we make an overview of the parameters that play a role in the energetic and kinetic aspects of the charge separation and recombination processes occurring in PyrDMA. These parameters are compiled in Table 8.

Table 8. The Gibbs free energy change for charge separation, the solvent $\left(\lambda_{s}\right)$ and internal reorganization energies $\left(\lambda_{i}\right)$ as well as the solvent properties ( $n$ and $\epsilon_{S}$ ). The internal reorganisation energies calculated using the four-point method with the TZ2P basis and the CAMY-B3LYP functional. CRS = charge recombination to the singlet ground state, CRT = charge recombination to the triplet state, $C S=$ charge separation.

\begin{tabular}{ccccccc}
\hline Solvent & $n$ & $\epsilon_{S}$ & $\begin{array}{c}\Delta G_{C S} \\
(\mathrm{eV})\end{array}$ & $\lambda_{s}(\mathrm{eV})$ & $\begin{array}{c}\lambda_{i, C R S} \\
(\mathrm{eV})\end{array}$ & $\begin{array}{c}\lambda_{i, C R T} \\
(\mathrm{eV})\end{array}$ \\
\hline Gas & 1.00 & 1.00 & -0.110 & 0.00000 & 0.7817 & 0.7571 \\
$\mathrm{NHX}$ & 1.37 & 1.88 & -0.178 & 0.00135 & 0.7618 & 0.7379 \\
$\mathrm{ACN}$ & 1.34 & 37.5 & -0.493 & 0.81600 & 0.7187 & 0.6965
\end{tabular}

The following inputs values are used: $r^{-}=3.98 \AA, r^{+}=3.70 \AA, R_{C}=6.50 \AA, E^{0}\left(D^{+} / D\right)=+0.76 \mathrm{eV}$ vs SCE in ACN, $E^{0}\left(A / A^{-}\right)=-2.09 \mathrm{eV}$ vs SCE in $A C N$, For pyrene: ${ }^{1} E_{00}=3.26 \mathrm{eV}$.

An important parameter from the Marcus theory ${ }^{5,6}$ that can be calculated is the reorganization energy ${ }^{58}(\lambda)$. The reorganization energy consists of an external $\left(\lambda_{s}\right)$ and internal $\left(\lambda_{i}\right)$ component.

$$
\lambda=\lambda_{i}+\lambda_{s}
$$

The focus is on calculating $\lambda_{i}$, which measures the change in energy that is needed to rearrange the molecule upon charge separation from a product to a reactant state. The reorganization energy for the electron donor $\left(\lambda_{D}\right)$ and the electron acceptor $\left(\lambda_{A}\right)$ of the molecules can be calculated with the fourpoint method. ${ }^{59}$

$$
\begin{gathered}
\lambda_{\text {int }}=\lambda_{D}+\lambda_{A} \\
2 \lambda_{A}=\left(E_{0}^{-}-E_{-}^{-}\right)+\left(E_{-}^{0}-E_{0}^{0}\right) \\
2 \lambda_{D}=\left(E_{0}^{+}-E_{+}^{+}\right)+\left(E_{+}^{0}-E_{0}^{0}\right)
\end{gathered}
$$


$E_{\mathrm{G}}^{\mathrm{S}}$ represents the energy of state with charge $\mathrm{S}$ at geometry $\mathrm{G} \cdot{ }^{60}$ For example $\mathrm{E}_{0}^{-}$is the energy of the anion at the optimized geometry of the neutral molecule (see also Appendix II).

The reorganization energy can be determined by calculating the different energies with the use of DFT. Four geometry optimizations need to be performed for the neutral fragments, the anionic, and cationic fragments (specifying the charges and unpaired electrons). Thereafter, four single point calculations need to be calculated. The best model compounds that can be used for PyrDMA are N,Ndimethylaniline for the donor and 1-methylpyrene for the acceptor. They were used to estimate the internal reorganization energies.

Calculated internal reorganization energies are presented Table 8. Clearly, a relatively large internal reorganization energy is observed. The internal reorganization energy of dimethylaniline has been calculated before. ${ }^{61}$ The normal mode analysis method resulted in 5400 and $3500 \mathrm{~cm}^{-1}(0.67$ and 0.43 $\mathrm{eV}$ ) giving an average of $0.55 \mathrm{eV}$. Other work ${ }^{62}$ resulted in values of 0.41 and $0.28 \mathrm{eV}$ (average of 0.345 eV) for N,N-dimethylaniline.

The solvent reorganizational energy $\lambda_{s}$ is defined by equation 8 .

$$
\lambda_{s}=\frac{e^{2}}{4 \pi \epsilon_{0}}\left(\left(\frac{1}{2 r^{+}}+\frac{1}{2 r^{-}}\right)-\frac{1}{R_{C}}\right)\left(\frac{1}{n^{2}}-\frac{1}{\epsilon_{s}}\right)
$$

$n$ here is the refractive index of the solvent. $\epsilon_{S}$ is the relative dielectric constant of the medium. $r^{+}$ and $r^{-}$are the cation and anion radii. ${ }^{64} R_{C}$ is the centre to centre distance between pyrene and dimethylaniline. $\epsilon_{0}$ is the vacuum permittivity, $\mathrm{n}$ the refractive index and $e$ the elementary electronic charge. ${ }^{66}$ The last parameter that is needed for the Marcus equation is the Gibbs energy $(\Delta G)$. The Gibbs free energy is the driving force for CS and the determines the rate for CS. The Gibbs energy equals $\Delta E$ and can be calculated by taking the difference in energy of the two states of interest. The excitation energies can in this case be taken to calculate the Gibbs energy. The Gibbs free energy for charge separation can also be estimated using the following equation.

$$
\Delta G_{C S}=e\left(E^{0}\left(D^{+} / D\right)-E^{0}\left(A / A^{-}\right)\right)-{ }^{1} \Delta E_{00}-\frac{e^{2}}{4 \pi \epsilon_{0} \epsilon_{S} R_{C}}
$$

The Gibbs free energy consists out of four terms: the energy it costs to oxidize the donor $E^{0}\left(D^{+} / D\right)$, the energy required to reduce the acceptor $E^{0}\left(A / A^{-}\right)$, how much (useable) energy is put into the system by excitation ${ }^{1} \Delta E_{00}$. This is the energy of the locally excited state $S_{2}$. A Coulomb term $\frac{e^{2}}{4 \pi \epsilon_{0} \epsilon_{S} R_{C}}$, 
which describes solvent effects. The oxidation potential of the DMA donor, and the reduction potential of the Pyrene acceptor, as well as its singlet state energy in ACN are well known. ${ }^{63,64}$

Polar solvents are better at stabilizing charged molecules, making charge separation more favourable in polar solvents. Changing to a different solvent requires a solvent correction term:

$$
\Delta G_{C S}=e\left(E^{0}\left(D^{+} / D\right)-E^{0}\left(A / A^{-}\right)\right)-{ }^{1} E_{00}-\frac{e^{2}}{4 \pi \epsilon_{0} \epsilon_{s} R_{C}}-\frac{e^{2}}{8 \pi \epsilon_{0}}\left(\frac{1}{r^{+}}+\frac{1}{r^{-}}\right)\left(\frac{1}{\epsilon_{E C}}-\frac{1}{\epsilon_{S}}\right)
$$

Using the Gibbs free energy for charge separation, the rates for charge separation can now be calculated. As observed in Table 8 the Gibbs free energy is highest in ACN and lowest in the gas phase, which implies that the driving force for charge separation is the highest in ACN.

If we apply the distance reported in Table 8 for the different states, a slight variation is introduced.

Table 9. The solvent reorganisation energy calculated from the centre to centre distance.

\begin{tabular}{cccc}
\hline Solvent & $\lambda_{s, C S}(\mathrm{eV})$ & $\lambda_{s, C R S}(\mathrm{eV})$ & $\lambda_{S, C R T}(\mathrm{eV})$ \\
\hline Gas & 0.000 & 0.000 & 0.000 \\
NHX & 0.0018 & 0.0018 & 0.0018 \\
ACN & 1.0294 & 0.9326 & 0.9326 \\
\hline
\end{tabular}

In Figure 14, the different energies of the states as well as the couplings are presented for the charge separation as well as for the charge recombination process for PyrDMA in NHX. 


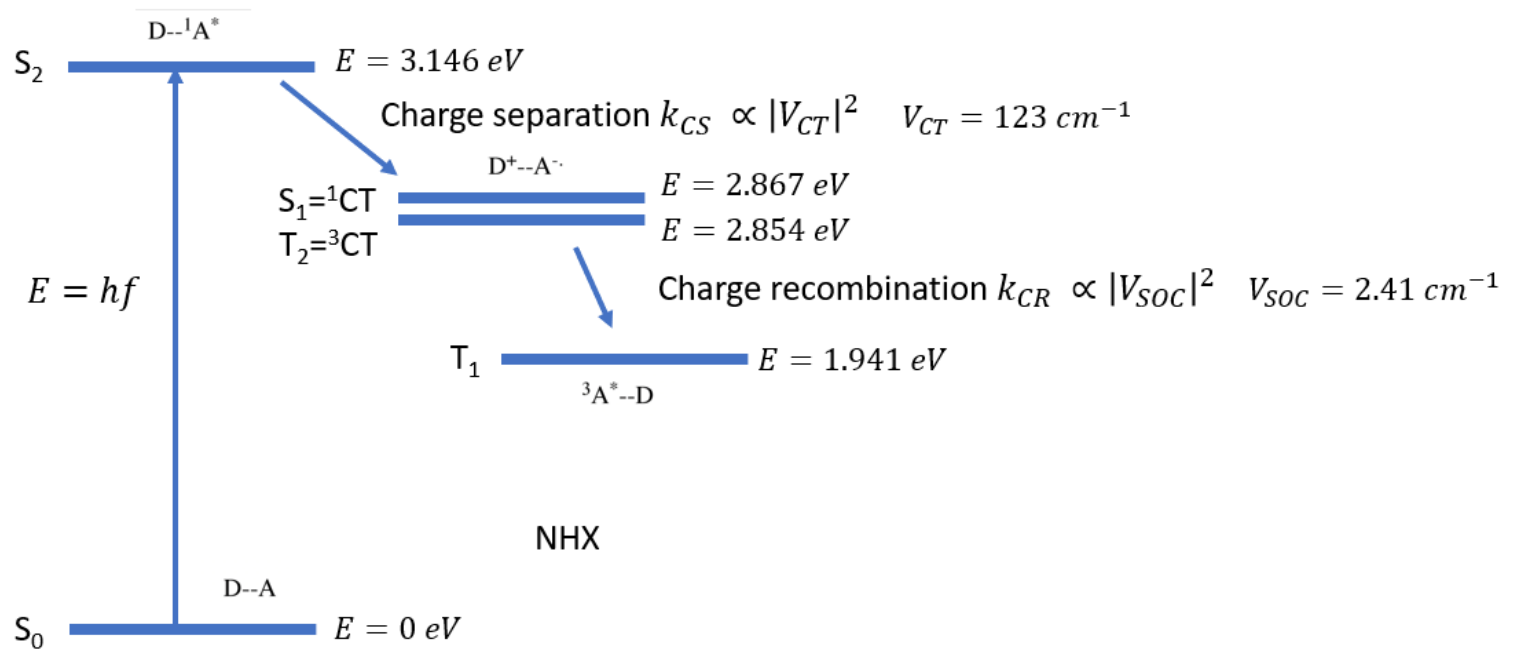

Figure 14. Energies of the excited states as well as the electronic coupling and spin orbit coupling of PyrDMA in n-hexane solvent as determined with ADF. The excited state energies are calculated with the TZP basis and PBE functional at the $S_{1}$ geometry, which in return is calculated with the TZ2P basis and PBE functional.

From these calculated numbers presented in Figure 14, we estimate a triplet emission at $640 \mathrm{~nm}$, the Gibbs free energy changes for charge separation $(-0.292 \mathrm{eV})$ and charge recombination to the triplet $(-0.913 \mathrm{eV})$. The energetic level of the triplet excited state of the pyrene can be estimated from the phosphorescence of pyrene or substituted pyrene. ${ }^{65,66}$ Emission maxima are reported at 647, 664 and $680 \mathrm{~nm}$ for the phosphorescence of substituted pyrene units. Thus, the triplet level is estimated to be between 1.91 and $1.82 \mathrm{eV}$. For pristine pyrene it is $2.1 \mathrm{eV},(2.08 \mathrm{eV}$ for 1-methylpyrene). The energy of the singlet state of pyrene is reported to be $3.34 \mathrm{eV}$ in nonpolar and $3.33 \mathrm{eV}$ in a polar medium. ${ }^{66}$

Classical Marcus Theory does not correctly predict the rates in the Marcus inverted regime. ${ }^{67}$ This deviation can be explained by quantum chemical vibrational effects that start to play a role. PESs of the two states at the minima can be described as vibrational normal modes that lead to vibrational eigenfunctions within a quantum mechanical description. The rates in this regime can be determined with the Marcus- Levich-Jortner theory to calculate the CS and CR rates. Therefore, we first set out to apply the single mode semi-classical Marcus equation, ${ }^{54}$ in which the electronic coupling is substituted by the spin orbit coupling: ${ }^{41}$

$$
k_{C R}=\frac{2 \pi^{3 / 2}}{h \sqrt{\lambda_{S} k_{B} T}}\left|V_{S O C}\right|^{2} \sum_{\mathrm{n}=0}^{\mathrm{m}} \frac{e^{-S} S^{n}}{n !} \exp \left(\frac{-\left(\Delta G+\lambda_{S}+n h \omega\right)^{2}}{4 \lambda_{S} k_{B} T}\right)
$$




$$
\begin{aligned}
& S_{e f f}=\sum_{i} S_{i} \\
& \omega_{e f f}=\sum_{i} \frac{\omega_{i} S_{i}}{S_{e f f}}
\end{aligned}
$$

With $\mathrm{S}_{\mathrm{i}}=\lambda_{i} / h \omega$. The factor $\mathrm{S}_{\text {eff }}$ is the Huang-Rhys factor of these effective modes. It is a measure of the strength of electron-phonon coupling. Upon photoexcitation, the equilibrium positions of the nuclei are displaced and the Huang-Rhys factor is associated with this phenomenon. With the individual Huang-Rhys factors $S_{i}$ for each vibrational mode, an effective Huang-Rhys factor can be calculated. $\omega_{\text {eff }}$ denotes an effective mode frequency, which can be calculated with the individual high frequency $\omega_{i}$ normal modes. ${ }^{68}$

Estimates for some of these parameters (for charge separation) have been reported for a 'donorinversed' molecule (P1D), in which the amino unit is para relative to the $\mathrm{CH}_{2} \operatorname{linker}\left(\lambda_{\mathrm{i}}=0.543 \mathrm{eV} ; \mathrm{S}=\right.$ $\left.0.65, h \omega=0.840 \mathrm{eV}, \omega=6775 \mathrm{~cm}^{-1}\right) .{ }^{69}$ The Gibbs free energy for electron transfer in acetonitrile was evaluated experimentally to be $-0.48 \mathrm{eV}$, and is also reported in this work.

However, the determination of S with DFT for PyrDMA remains pending for the future, as the values obtained for PyrDMA so-far were very low (too low), which we assume to be due to the large conformational changes between the charge transfer state and the triplet state.

Having determined the $V_{s o c}$ value $\left(2.46 \mathrm{~cm}^{-1}\right)$ as well as the solvent reorganization energy $\left(\lambda_{s}=0.00135\right.$ $\mathrm{eV})$ in NHX at the optimized geometry, we can now estimate the first part of the rate $\left(k_{\mathrm{CR}}\right)$ with the Classical Marcus equation (see Table 10).

$$
k_{C R}=\frac{2 \pi^{3 / 2}}{h \sqrt{\lambda k_{B} T}}\left|V_{S O C}\right|^{2} * \exp \left(\frac{-(\Delta G+\lambda)^{2}}{4 \lambda k_{B} T}\right)
$$

Clearly a reasonable correlation between theoretical and experimental rates is observed, with just one order of magnitude difference. The situation is similar for the rate for the charge separation (5.9 $\left.\times 10^{11} \mathrm{~s}^{-1}\right)$.

Clearly, the charge recombination to the ground state $\left(7.89 \times 10^{6} \mathrm{~s}^{-1}\right)$ is strongly underestimated by the Classical Marcus theory, due to inverted region effects. An overview is given in Table 10. 
It has to be noted that we apply two different $V_{\text {soc }}$ values $\left(2.47 \mathrm{~cm}^{-1}\right.$ and $V_{\text {soc }}$ value $3.22 \mathrm{~cm}^{-1}$, the highest values in Table 2).

Likewise, two different $\Delta \mathrm{G}$ values for $\mathrm{CR}_{\mathrm{S}}(-3.08$ and $-2.87 \mathrm{eV})$ and for charge separation (CS) from S2 to S1, (-0.178 and $-0.49 \mathrm{eV})$, with either computational source (see figure 14) or based on experimental approximations (Table 8) are applied. By using computational or experimental energetics we are able to correlate the Classsical Marcus model to the experimental rates.

Table 10. Parameters for rate determination at $T=295 \mathrm{~K}$ in $n$-hexane (NHX).

\begin{tabular}{lccccccc}
\hline & $\begin{array}{c}\Delta G \\
(\mathrm{eV})\end{array}$ & $\begin{array}{c}\lambda_{i} \\
(\mathrm{eV})\end{array}$ & $\begin{array}{c}\lambda_{s} \\
(\mathrm{eV})\end{array}$ & $\begin{array}{c}V \\
\left(\mathrm{~cm}^{-1}\right)\end{array}$ & $\begin{array}{c}k_{\text {opt }} \\
\left(\mathrm{s}^{-1}\right)\end{array}$ & $\begin{array}{c}k_{\text {calc }} \\
\left(\mathrm{s}^{-1}\right)\end{array}$ & $\begin{array}{c}k_{\text {exp }} \\
\left(\mathrm{s}^{-1}\right)\end{array}$ \\
\hline $\boldsymbol{C} \boldsymbol{R}_{T}$ & -0.73 & 0.7379 & 0.00135 & 2.47 & $1.84 \times 10^{9}$ & $1.84 \times 10^{9}$ & $2.50 \times 10^{10}$ \\
$\boldsymbol{C} \boldsymbol{R}_{T}$ & -0.73 & 0.7379 & 0.00135 & 3.22 & $3.13 \times 10^{9}$ & $3.12 \times 10^{9}$ & $2.50 \times 10^{10}$ \\
\hline $\boldsymbol{C} \boldsymbol{R}_{S}$ & -3.08 & 0.7618 & 0.00135 & 159 & $7.84 \times 10^{12}$ & $2.75 \times 10^{-22}$ & $7.89 \times 10^{6}$ \\
$\boldsymbol{C} \boldsymbol{R}_{S}$ & -2.87 & 0.7618 & 0.00135 & 159 & $7.50 \times 10^{12}$ & $1.29 \times 10^{-10}$ & $7.89 \times 10^{6}$ \\
\hline $\boldsymbol{C S}$ & -0.178 & 0.7000 & 0.00135 & 123.2 & $4.71 \times 10^{12}$ & $1.01 \times 10^{11}$ & $5.9 \times 10^{11}$ \\
$\boldsymbol{C S}$ & -0.49 & 0.7000 & 0.00135 & 123.2 & $4.71 \times 10^{12}$ & $2.52 \times 10^{12}$ & $5.9 \times 10^{11}$
\end{tabular}

$126.7 \mathrm{~ns}$ is the exciplex lifetime in NHX, for intermolecular interaction, ${ }^{70}$ the following energy values were taken ${ }^{1} E_{00}=3.26$ $\mathrm{eV},{ }^{1} E_{C T}=2.87 \mathrm{eV},{ }^{3} E_{00}=2.14 \mathrm{eV}$, next to the values in Table 6 and 8.

\section{$4 \quad$ Conclusions and Future Outlook}

The dependence of the SOCME as well as $V_{C T}$ as a function of the dihedral angle between the donor and acceptor have been determined using (TD-)DFT. The opposing effects, maximum at $\sim 90^{\circ}$ of the SOCME and minimum of $V_{C T}$ at that same angle fit perfectly within the current paradigms. At optimized geometries of the locally excited state and singlet charge transfer state (PBE/TZP), values of $V_{S O C}=2.47 \mathrm{~cm}^{-1}$ and $V_{C T}=123.2 \mathrm{~cm}^{-1}$ are found in NHX. In ACN, the values at the optimized geometries are $V_{S O C}=2.56 \mathrm{~cm}^{-1}$ and $V_{C T}=96.4 \mathrm{~cm}^{-1}$. Using these values, the calculated rates for triplet charge recombination are close to the experimental charge recombination rates in NHX. The relatively large amplitude motions of the donor unit that occur after photoexcitation $\left(S_{2} \rightarrow S_{1} \rightarrow T_{1}\right.$ in 40 ps), together with the time scales, indicate that PyrDMA acts as a molecular "fin", giving a fast push followed by a slow back relaxation ( $\mu$ s timescale triplet decay) to its original location. 
The main message of this work is that the ratios of the optimal rates of charge separation and charge recombination to the triplet as well as the ratios of the optimal rates for charge recombination to the triplet and charge recombination to the singlet are governed by their respective couplings, which all are very sensitive to slight conformational changes. These ratios need to be optimal in order for the SOCT-ISC mechanism to operate efficiently, leading to high triplet yields. Clearly, there are examples of molecular systems in which all these aspects are optimal.

SOCME values for acetone $\left(\sim 62 \mathrm{~cm}^{-1}\right)$ and for 4-thio-thymine $\left(\sim 157 \mathrm{~cm}^{-1}\right)$ can be reproduced from literature very well. The SOC values are very dependent on the geometry that is used, but not so much on the XC. LB94 gives the highest values. A long-range parameter of $0.34(\gamma)$ is appropriate for the large range of excited-state geometries, that we studied in this work.

The impression is that PBE is more appropriate for geometry optimization (leading to less extreme conformations) and that CAM-B3LYP is more suited to calculate properties at single point geometries. However, more elaborate studies with different electron donor-acceptor molecules may be performed to substantiate this argument. The excitation energies obtained with ADF can be correlated well with the experimentally available quantities. The procedure to use ADF to calculate and predict properties with respect to the SOCT-ISC mechanism should be generalized to other molecules. Future computational studies should emphasize and substantiate the relevance of all aspects for the proposed method. Computationally obtained results need to be compared with experimental findings and this method needs to be optimized where needed. ${ }^{71,72}$ 
Author Contributions: Conceptualization by R.M.W. and D.J.G.; all DFT calculations as well as the analysis and visualization of the output data by S.B.; writing-original draft preparation based on concept report of MSc internship of S.B. at the Molecular Photonics group at UvA as well as the literature thesis of D.M.E.v.R. (including Appendix II and III) within the MSc program Molecular Sciences at UvA; Final writing, review and editing by S.B., D.J.G and R.M.W.; supervision by R.M.W.

\section{Acknowledgements}

This project has received funding from the European Union's Horizon 2020 research and innovation programme under the Marie Sklodowska-Curie grant agreement no. 764837. We thank the Universiteit van Amsterdam for structural support. This work was carried out on the Dutch national e-infrastructure with the support of SURF Cooperative.

\section{Supporting Information}

Representative ADF input and output files (SI-1.zip) are provided as well as an extensive description of the procedures with (repeated) references (SI-2.pdf). Coordinate files for the various excited states are supplied as mol2 files. Matlab files for data analysis and input file generation, Mathematica modules to calculate the rates are provided as cdf and notebook files (SI-1.zip). 


\begin{tabular}{|c|c|}
\hline TD-DFT & Time-dependent density functional theory \\
\hline ADF & Amsterdam Density Functional \\
\hline DMA & dimethylaniline \\
\hline Pyr & pyrene \\
\hline NHX & n-hexane \\
\hline $\mathrm{ACN}$ & acetonitrile \\
\hline CS & charge separation \\
\hline CR & charge recombination \\
\hline ISC & Intersystem crossing \\
\hline CT & Charge transfer \\
\hline SO-ISC & Spin-orbit coupling intersystem crossing \\
\hline SOCT-ISC & Spin-orbit charge transfer intersystem crossing \\
\hline MO & Molecular orbital \\
\hline SOC & Spin-orbit coupling \\
\hline SOCME & Spin-orbit coupling matrix element \\
\hline GS & Ground state \\
\hline LE & Local excitation \\
\hline $\mathrm{T}_{\mathrm{n}}$ & triplet (state) \\
\hline$S_{n}$ & Singlet (state) \\
\hline${ }^{\mathrm{n}} \mathrm{CS}$ & Charge separated (state) \\
\hline$V_{C T}$ & Electronic coupling \\
\hline$V_{S O C}$ & Spin orbit coupling \\
\hline$T$ & Temperature \\
\hline$\hbar$ & reduced Planck constant \\
\hline$k_{B}$ & Boltzmann constant \\
\hline$c$ & Speed of light \\
\hline$\lambda$ & Reorganization Energy \\
\hline$\tau$ & lifetime of a state \\
\hline PES & Potential Energy Surface \\
\hline$\Delta G$ & Gibbs free energy \\
\hline$S$ & Huang Rhys factor \\
\hline FCWD & Frank Condon Weighted Density \\
\hline$f_{S O C}$ & Oscillator strength \\
\hline TDA & Tamm-Dancoff approximation \\
\hline NTO & Natural Transition Orbital \\
\hline cosmo & Conductor-like screening model \\
\hline ZORA & Zero-order regular approximation \\
\hline
\end{tabular}




\section{References}

${ }^{1}$ Turro, N. J.; Ramamurthy, V.; Scaiano, J. C. Principles of Molecular Photochemistry: An Introduction; University Science Books, 2009.

${ }^{2}$ Marian, C. M. Spin-Orbit Coupling and Intersystem Crossing in Molecules. Wiley Interdiscip. Rev. Comput. Mol. Sci. 2012, 2 (2), 187-203. https://doi.org/10.1002/wcms.83.

${ }^{3}$ Gao, X.; Bai, S.; Fazzi, D.; Niehaus, T.; Barbatti, M.; Thiel, W. Evaluation of Spin-Orbit Couplings with LinearResponse Time-Dependent Density Functional Methods. J. Chem. Theory Comput. 2017, 13 (2), 515-524. https://doi.org/10.1021/acs.jctc.6b00915.

${ }^{4}$ Hou, Y.; Biskup, T.; Rein, S.; Wang, Z.; Bussotti, L.; Russo, N.; Foggi, P.; Zhao, J.; Di Donato, M.; Mazzone, G.; et al. Spin-Orbit Charge Recombination Intersystem Crossing in Phenothiazine-Anthracene Compact Dyads: Effect of Molecular Conformation on Electronic Coupling, Electronic Transitions, and Electron Spin Polarizations of the Triplet States. J. Phys. Chem. C. 2018, 122 (49), 27850-27865. https://doi.org/10.1021/acs.jpcc.8b08965.

${ }^{5}$ Marcus, R. A. On the Theory of Electron-Transfer Reactions. VI. Unified Treatment for Homogeneous and Electrode Reactions. J. Chem. Phys. 1965, 43 (2), 679-701. https://doi.org/10.1063/1.1696792.

${ }^{6}$ Marcus, R. A. Electron Transfer Reactions in Chemistry: Theory and Experiment (Nobel Lecture). Angew. Chem. Int. Ed. Engl. 1993, 32 (8), 1111-1121. https://doi.org/10.1002/anie.199311113.

${ }^{7}$ Monti, A.; De Groot, H. J. M.; Buda, F. In-Silico Design of a Donor-Antenna-Acceptor Supramolecular Complex for Photoinduced Charge Separation. J. Phys. Chem. C. 2014, 118 (29), 15600-15609.

https://doi.org/10.1021/jp505105a.

${ }^{8}$ Hou, Y.; Zhang, X.; Chen, K.; Liu, D.; Wang, Z.; Liu, Q.; Zhao, J.; Barbon, A. Charge Separation, Charge Recombination, Long-Lived Charge Transfer State Formation and Intersystem Crossing in Organic Electron Donor/Acceptor Dyads. J. Mater. Chem. C. 2019, 7 (39), 12048-12074. https://doi.org/10.1039/c9tc04285g.

${ }^{9}$ Gibbons, D. J.; Farawar, A.; Mazzella, P.; Leroy-Lhez, S.; Williams, R. M. Making Triplets from Photo-Generated Charges: Observations, Mechanisms and Theory. Photochem. Photobiol. Sci. 2020, 19 (2), 136-158.

https://doi.org/10.1039/c9pp00399a.

${ }^{10}$ Schultz, D. M.; Yoon, T. P. Solar Synthesis: Prospects in Visible Light Photocatalysis. Science (80). 2014, 343 (6174). https://doi.org/10.1126/science.1239176.

${ }^{11}$ Smith, M. B.; Michl, J. Singlet Fission. Chem. Rev. 2010, 110 (11), 6891-6936.

https://doi.org/10.1021/cr1002613.

${ }^{12}$ Celli, J. P.; Spring, B. Q.; Rizvi, I.; Evans, C. L.; Samkoe, K. S.; Verma, S.; Pogue, B. W.; Hasan, T. Imaging and Photodynamic Therapy: Mechanisms, Monitoring, and Optimization. Chem. Rev. 2010, 110 (5), 2795-2838. https://doi.org/10.1021/cr900300p.

13 Üngördü, A. Electronic, Optical, and Charge Transfer Properties of Porphyrin and Metallated Porphyrins in Different Media. Int. J. Quantum Chem. 2020, 120 (6). https://doi.org/10.1002/qua.26128.

${ }^{14}$ Ronca, E.; De Angelis, F.; Fantacci, S. Time-Dependent Density Functional Theory Modeling of Spin-Orbit Coupling in Ruthenium and Osmium Solar Cell Sensitizers. J. Phys. Chem. C. 2014, 118 (30), 17067-17078. https://doi.org/10.1021/jp500869r.

${ }^{15}$ Tsvirko, M., Solovjev, K., Gradyushko, A., Dvornikov, S. Phosphorescence of Porphyrin Free Bases and Their Complexes with Light Metals. Opt. Spectrosc. 1975, 38, 400.

${ }^{16}$ Zhao, J.; Wu, W.; Sun, J.; Guo, S. Triplet Photosensitizers: From Molecular Design to Applications. Chem. Soc. Rev. 2013, 42 (12), 5323-5351. https://doi.org/10.1039/c3cs35531d.

${ }^{17}$ Yang, B.; Huang, S.; Luo, S. A. Theoretical Research on Intersystem Crossing, Radiative and Nonradiative Rates of Cyclometalated Platinum(II) Complexes. Theor. Chem. Acc. 2019, 138 (6), 77. https://doi.org/10.1007/s00214-019-2466-6.

${ }^{18}$ Minaev, B.; Ågren, H. Theoretical DFT Study of Phosphorescence from Porphyrins. Chem. Phys. 2005, 315 (3), 215-239. https://doi.org/10.1016/j.chemphys.2005.04.017.

${ }^{19}$ Filatov, M. A. Heavy-Atom-Free BODIPY Photosensitizers with Intersystem Crossing Mediated by Intramolecular Photoinduced Electron Transfer. Org. Biomol. Chem. 2019, 18 (1), 10-27.

https://doi.org/10.1039/c9ob02170a.

${ }^{20}$ Lv, M.; Yu, Y.; Sandoval-Salinas, M. E.; Xu, J.; Lei, Z.; Casanova, D.; Yang, Y.; Chen, J. Engineering the ChargeTransfer State to Facilitate Spin-Orbit Charge Transfer Intersystem Crossing in Spirobis[Anthracene]Diones. Angew. Chemie - Int. Ed. 2020, 59, 2-8. https://doi.org/10.1002/anie.202009439. 
${ }^{21}$ El-Sayed, M. A. Spin-Orbit Coupling and the Radiationless Processes in Nitrogen Heterocyclics. J. Chem. Phys 1963, 38, 2834. https://doi.org/10.1063/1.1733610.

22 Okada, T.; Karaki, I.; Matsuzawa, E.; Mataga, N.; Sakata, Y.; Misumi, S. Ultrafast Intersystem Crossing in Some Intramolecular Heteroexcimers. J. Phys. Chem. 1981, 85 (26), 3957-3960.

https://doi.org/10.1021/j150626a002.

${ }^{23}$ Van Willigen, H; Guilford Jones, I.; Farahat, M. S. Time-Resolved EPR Study of Photoexcited Triplet-State Formation in Electron-Donor-Substituted Acridinium Ions. J. Phys. Chem. 1996, 100 (9), 3312-3316.

https://doi.org/10.1021/JP953176+.

${ }^{24}$ Dance, Z. E. X.; Mickley, S. M.; Wilson, T. M.; Ricks, A. B.; Scott, A. M.; Ratner, M. A.; Wasielewski, M. R. Intersystem Crossing Mediated by Photoinduced Intramolecular Charge Transfer: Julolidine - Anthracene Molecules with Perpendicular $\pi$ Systems. J. Phys. Chem. A. 2008, 112 (18), 4194-4201.

https://doi.org/10.1021/jp800561g.

${ }^{25}$ SCM. Amsterdam Modeling Suite Making Computational Chemistry Work For You Software for Chemistry \& Materials https://www.scm.com/ (accessed Feb 9, 2021).

${ }^{26}$ Buck, J. T.; Boudreau, A. M.; DeCarmine, A.; Wilson, R. W.; Hampsey, J.; Mani, T. Spin-Allowed Transitions Control the Formation of Triplet Excited States in Orthogonal Donor-Acceptor Dyads. Chem. 2019, 5 (1), 138155. https://doi.org/10.1016/j.chempr.2018.10.001.

${ }^{27}$ Sasikumar, D.; John, A. T.; Sunny, J.; Hariharan, M. Access to the Triplet Excited States of Organic Chromophores. Chem. Soc. Rev. 2020, 49 (17), 6122-6140. https://doi.org/10.1039/d0cs00484g.

${ }^{28}$ Nguyen, V.-N.; Yan, Y.; Zhao, J.; Yoon, J. Heavy-Atom-Free Photosensitizers: From Molecular Design to Applications in the Photodynamic Therapy of Cancer. Acc. Chem. Res. 2021, 54 (1), 207-220. https://doi.org/10.1021/acs.accounts.0c00606.

${ }^{29}$ Sutton, C.; Sears, J. S.; Coropceanu, V.; Brédas, J. L. Understanding the Density Functional Dependence of DFT-Calculated Electronic Couplings in Organic Semiconductors. J. Phys. Chem. Lett. 2013, 4 (6), $919-924$. https://doi.org/10.1021/jz3021292.

${ }^{30}$ Pye, C. C.; Ziegler, T. An Implementation of the Conductor-like Screening Model of Solvation within the Amsterdam Density Functional Package. Theor. Chem. Acc. 1999, 101 (6), 396-408. https://doi.org/10.1007/s002140050457.

${ }^{31}$ Lowry, T. H.; Richardson, K. S.; Mechanism and theory in organic chemistry. New York : Harper \& Row, 1987

${ }^{32}$ Williams, R. M.; Zwier, J. M.; Verhoeven, J. W. Photoinduced Intramolecular Electron Transfer in a Bridged C60 (Acceptor)-Aniline (Donor) System; Photophysical Properties of the First “Active" Fullerene Diad. J. Am. Chem. Soc. 1995, 117 (14), 4093-4099. https://doi.org/10.1021/ja00119a025.

${ }^{33}$ Williams, R. M.; Koeberg, M.; Lawson, J. M.; An, Y.-Z.; Rubin, Y.; Paddon-Row, M. N.; Verhoeven, J. W. Photoinduced Electron Transfer to $\mathrm{C}_{60} \mathrm{C}_{60}$ across Extended 3- and 11-Bond Hydrocarbon Bridges: Creation of a Long-Lived Charge-Separated State. J. Org. Chem. 1996, 61 (15), 5055-5062.

https://doi.org/10.1021/jo960678q.

${ }^{34}$ Williams, R. M.; Verhoeven, J. W. Fluorescence of Fullerene-C70 and Its Quenching by Long-Range Intermolecular Electron Transfer. Chem. Phys. Lett. 1992, 194 (4), 6.

${ }^{35}$ te Velde, G.; Bickelhaupt, F. M.; Baerends, E. J.; Fonseca Guerra, C.; van Gisbergen, S. J. A.; Snijders, J. G.; Ziegler, T. Chemistry with ADF. J. Comput. Chem. 2001, 22, 931-967.

${ }^{36}$ Fonseca Guerra, C.; Snijders, J. G.; te Velde, G.; Baerends, E. J. Towards an order-N DFT method. Theor. Chem. Acc. 1998, 99, 391-403.

${ }^{37}$ van Lenthe, E.; Ehlers, A.; Baerends, E.-J. Geometry optimizations in the zero order regular approximation for relativistic effects. J. Chem. Phys. 1999, 110, 8943-8953.

${ }^{38}$ Pettersen, E. F.; Goddard, T. D.; Huang, C. C.; Couch, G. S.; Greenblatt, D. M.; Meng, E. C.; Ferrin, T. E. UCSF Chimera - A visualization system for exploratory research and analysis. J. Comput. Chem. 2004, 25, 1605-1612.

${ }^{39}$ Beljonne, D.; Shuai, Z.; Pourtois, G.; Bredas, J. L. Spin-Orbit Coupling and Intersystem Crossing in Conjugated Polymers: A Configuration Interaction Description. J. Phys. Chem. A. 2001, 105 (15), 3899-3907. https://doi.org/10.1021/jp010187w.

${ }^{40}$ Zhu, Q.; Feng, S.; Guo, X.; Chen, X.; Zhang, J. Strategy for Tuning the Up-Conversion Intersystem Crossing Rates in a Series of Organic Light-Emitting Diodes Emitters Relevant for Thermally Activated Delayed Fluorescence. Spectrochim. Acta - Part A Mol. Biomol. Spectrosc. 2019, 221, 117214.

https://doi.org/10.1016/j.saa.2019.117214. 
${ }^{41}$ Samanta, P. K.; Kim, D.; Coropceanu, V.; Brédas, J. L. Up-Conversion Intersystem Crossing Rates in Organic Emitters for Thermally Activated Delayed Fluorescence: Impact of the Nature of Singlet vs Triplet Excited States. J. Am. Chem. Soc. 2017, 139 (11), 4042-4051. https://doi.org/10.1021/jacs.6b12124.

42 Sun, H.; Hu, Z.; Zhong, C.; Chen, X.; Sun, Z.; Brédas, J. L. Impact of Dielectric Constant on the Singlet-Triplet Gap in Thermally Activated Delayed Fluorescence Materials. J. Phys. Chem. Lett. 2017, 8 (11), 2393-2398. https://doi.org/10.1021/acs.jpclett.7b00688.

${ }^{43}$ Cardeynaels, T.; Paredis, S.; Deckers, J.; Brebels, S.; Vanderzande, D.; Maes, W.; Champagne, B. Finding the Optimal Exchange-Correlation Functional to Describe the Excited State Properties of Push-Pull Organic Dyes Designed for Thermally Activated Delayed Fluorescence. Phys. Chem. Chem. Phys. 2020, 22 (28), 16387-16399. https://doi.org/10.1039/D0CP02409K.

${ }^{44}$ Hu, Y.; Hou, Y.; Wang, Z.; Li, Y.; Zhao, J. 3,5-Anthryl-Bodipy Dyad/Triad: Preparation, Effect of F-B-F Induced Conformation Restriction on the Photophysical Properties, and Application in Triplet-Triplet-Annihilation Upconversion. J. Chem. Phys. 2020, 153 (22), 224304. https://doi.org/10.1063/5.0025224.

${ }^{45}$ Martin, R. L. Natural Transition Orbitals. J. Chem. Phys. 2003, 118, 4775. https://doi.org/10.1063/1.1558471.

${ }^{46}$ Gonzalez-Vazquez, J. P.; Burn, P. L.; Powell, B. J. Interplay of Zero-Field Splitting and Excited State Geometry Relaxation in Fac-Ir(Ppy)3. Inorg. Chem. 2015, 54 (21), 10457-10461.

https://doi.org/10.1021/acs.inorgchem.5b01918.

${ }^{47}$ Chen, K.; Zhao, J.; Li, X.; Gurzadyan, G. G. Anthracene-Naphthalenediimide Compact Electron Donor/Acceptor Dyads: Electronic Coupling, Electron Transfer, and Intersystem Crossing. J. Phys. Chem. A. 2019, 123 (13), 25032516. https://doi.org/10.1021/acs.jpca.8b11828.

${ }^{48}$ Wang, Z.; Sukhanov, A. A.; Toffoletti, A.; Sadiq, F.; Zhao, J.; Barbon, A.; Voronkova, V. K.; Dick, B. Insights into the Efficient Intersystem Crossing of Bodipy-Anthracene Compact Dyads with Steady-State and Time-Resolved Optical/Magnetic Spectroscopies and Observation of the Delayed Fluorescence. J. Phys. Chem. C. 2019, 123 (1), 265-274. https://doi.org/10.1021/acs.jpcc.8b10835.

${ }^{49}$ Letrun, R.; Lang, B.; Yushchenko, O.; Wilcken, R.; Svechkarev, D.; Kolodieznyi, D.; Riedle, E.; Vauthey, E. Excited-State Dynamics of a Molecular Dyad with Two Orthogonally-Oriented Fluorophores. Phys. Chem. Chem. Phys. 2018, 20 (48), 30219-30230. https://doi.org/10.1039/c8cp05356a.

${ }^{50}$ Liu, T.; Troisi, A. Absolute Rate of Charge Separation and Recombination in a Molecular Model of the P3HT/PCBM Interface. J. Phys. Chem. C. 2011, 115 (5), 2406-2415. https://doi.org/10.1021/jp109130y.

${ }^{51}$ Wen, S. H.; Li, A.; Song, J.; Deng, W. Q.; Han, K. L.; Goddard, W. A. First-Principles Investigation of Anistropic Hole Mobilities in Organic Semiconductors. J. Phys. Chem. B 2009, 113 (26), 8813-8819.

https://doi.org/10.1021/jp900512s.

52 Martínez, J. P.; Trujillo-González, D. E.; Götz, A. W.; Castillo-Alvarado, F. L.; Rodríguez, J. I. Effects of Dispersion Forces on Structure and Photoinduced Charge Separation in Organic Photovoltaics. J. Phys. Chem. C. 2017, 121 (37), 20134-20140. https://doi.org/10.1021/acs.jpcc.7b05107.

${ }^{53}$ Kelber, J. B.; Panjwani, N. A.; Wu, D.; Gómez-Bombarelli, R.; Lovett, B. W.; Morton, J. J. L.; Anderson, H. L. Synthesis and Investigation of Donor-Porphyrin-Acceptor Triads with Long-Lived Photo-Induced ChargeSeparate States. Chem. Sci. 2015, 6 (11), 6468-6481. https://doi.org/10.1039/C5SC01830G.

${ }^{54}$ Chaudhuri, S.; Hedström, S.; Méndez-Hernández, D. D.; Hendrickson, H. P.; Jung, K. A.; Ho, J.; Batista, V. S. Electron Transfer Assisted by Vibronic Coupling from Multiple Modes. J. Chem. Theory Comput. 2017, 13 (12), 6000-6009. https://doi.org/10.1021/acs.jctc.7b00513.

${ }^{55}$ Peach, M. J. G.; Benfield, P.; Helgaker, T.; Tozer, D. J. Excitation Energies in Density Functional Theory: An Evaluation and a Diagnostic Test. J. Chem. Phys. 2008, 128 (4), 044118. https://doi.org/10.1063/1.2831900.

${ }^{55}$ Marcus, R. A. Electron Transfer Reactions in Chemistry: Theory and Experiment (Nobel Lecture). Angew. Chem. Int. Ed. Engl. 1993, 32 (8), 1111-1121. https://doi.org/10.1002/anie.199311113.

${ }^{56}$ Plasser, F.; Lischka, H. Analysis of Excitonic and Charge Transfer Interactions from Quantum Chemical Calculations. J. Chem. Theory Comput. 2012. https://doi.org/10.1021/ct300307c.

${ }^{57}$ Dreuw, A.; Head-Gordon, M. Failure of Time-Dependent Density Functional Theory for Long-Range ChargeTransfer Excited States: The Zinc bacteriochlorin-Bacteriochlorin and Bacteriochlorophyll-Spheroidene Complexes. J. Am. Chem. Soc. 2004, 126 (12), 4007-4016. https://doi.org/10.1021/ja039556n.

${ }^{58}$ D'Souza, F.; Subbaiyan, N. K.; Xie, Y.; Hill, J. P.; Ariga, K.; Ohkubo, K.; Fukuzumi, S. Anion-ComplexationInduced Stabilization of Charge Separation. J. Am. Chem. Soc. 2009, 131 (44), 16138-16146. https://doi.org/10.1021/ja9048306. 
${ }^{59}$ Nelsen, S. F.; Blackstock, S. C.; Kim, Y. Estimation of Inner Shell Marcus Terms for Amino Nitrogen Compounds by Molecular Orbital Calculations. J. Am. Chem. Soc. 1987, 109 (3), 677-682. https://doi.org/10.1021/ja00237a007.

${ }^{60}$ Daswani, U.; Singh, U.; Sharma, P.; Kumar, A. From Molecules to Devices: A DFT/TD-DFT Study of Dipole Moment and Internal Reorganization Energies in Optoelectronically Active Aryl Azo Chromophores. J. Phys. Chem. C. 2018, 122 (26), 14390-14401. https://doi.org/10.1021/acs.jpcc.8b04070.

${ }^{61}$ Scherer, P. O. J. Intramolecular Reorganization of the Electron Donor $N, N$-Dimethylaniline. J. Phys. Chem. A 2003, 107 (40), 8327-8329. https://doi.org/10.1021/jp027855d.

${ }^{62}$ Scherer, P. O. J.; Tachiya, M. Computer Simulation Studies of Electron Transfer Parameters for Cyanoanthracene/N,N-Dimethylaniline Solutions. J. Chem. Phys. 2003, 118 (9), 4149-4156. https://doi.org/10.1063/1.1541617.

${ }^{63}$ Schomburg, H.; Staerk, H.; Weller, A. Electron Transfer Reactions And Inhibition Of Triplet State Formation In Mlxed Fluorescence Quencher ExperIments. Chem. Phys. Lett.., 22 (1973), p. 1

${ }^{64}$ Vân Anh, N.; Schlosser, F.; Groeneveld, M. M.; van Stokkum, I. H. M.; Würthner, F.; Williams, R. M. Photoinduced Interactions in a Pyrene-Calix[4]Arene-Perylene Bisimide Dye System: Probing Ground-State Conformations with Excited-State Dynamics of Charge Separation and Recombination. J. Phys. Chem. C. 2009, 113 (42), 18358-18368. https://doi.org/10.1021/jp9055279.

${ }^{65}$ Leroy-lhez, S.; Belin, C.; D’aleo, A.; Williams, R. M.; De Cola, L.; Fages, F. Extending Excited-State Lifetimes by Interchromophoric Triplet-State Equilibration in a Pyrene-Ru(II)Diimine Dyad System. Supramol. Chem. 2003, 15 (7-8), 627-637. https://doi.org/10.1080/10610270310001605214

${ }^{66}$ Montalti, M.; Credi, A.; Prodi, L.; Gandolfi, M. T. Handbook of Photochemistry; CRC Press, 2006.

${ }^{67}$ Verhoeven, J. W.; Van Ramesdonk, H. J.; Groeneveld, M. M.; Benniston, A. C.; Harriman, A. Long-Lived Charge-Transfer States in Compact Donor-Acceptor Dyads. ChemPhysChem. 2005, 6(11), 2251-2260. https://doi.org/10.1002/cphc.200500029.

${ }^{68}$ Ruhoff, P. T.; Ratner, M. A. Algorithms for Computing Franck-Condon Overlap Integrals. Int. J. Quantum Chem. 2000, 77 (1), 383-392. https://doi.org/10.1002/(SICI)1097-461X(2000)77:1<383::AID-QUA38>3.0.CO;20 .

${ }^{69}$ Rujkorakarn, R.; Tanaka, F. Three-Dimensional Representations of Photo-Induced Electron Transfer Rates in Pyrene-(CH2)n-N, N'-Dimethylaniline Systems Obtained by Three Electron Transfer Theories. J. Mol. Graph. Model. 2009, 27 (5), 571-577. https://doi.org/10.1016/j.jmgm.2008.09.008.

${ }^{70}$ Werner, H.; Staerk, H.; Weller, A. Solvent, Isotope, and Magnetic Field Effects in the Geminate Recombination of Radical Ion Pairs. J. Chem. Phys. 1978, 68 (5), 2419-2426. https://doi.org/10.1063/1.436013. ${ }^{71}$ Imran, M.; Sukhanov, A. A.; Wang, Z.; Karatay, A.; Zhao, J.; Mahmood, Z.; Elmali, A.; Voronkova, V. K.; Hayvali, M.; Xing, Y. H.; et al. Electronic Coupling and Spin-Orbit Charge-Transfer Intersystem Crossing in PhenothiazinePerylene Compact Electron Donor/Acceptor Dyads. J. Phys. Chem. C. 2019, 123 (12), 7010-7024. https://doi.org/10.1021/acs.jpcc.8b12040.

72 Isukapalli, S. V. K.; Lekshmi, R. S.; Samanta, P. K.; Vennapusa, S. R. Formation of Excited Triplet States in Naphthalene Diimide and Perylene Diimide Derivatives: A Detailed Theoretical Study. J. Chem. Phys. 2020, 153 (12), 124301. https://doi.org/10.1063/5.0012476. 